\title{
BROUWERIAN SEMILATTICES
}

\author{
BY \\ PETER KÖHLER ${ }^{1}$
}

\begin{abstract}
Let $\mathbf{P}$ be the category whose objects are posets and whose morphisms are partial mappings $\alpha: P \rightarrow Q$ satisfying

(i) $\forall p, q \in \operatorname{dom} \alpha[p<q \Rightarrow \alpha(p)<\alpha(q)]$ and

(ii) $\forall p \in \operatorname{dom} \alpha \forall q \in Q[q<\alpha(p) \Rightarrow \exists r \in \operatorname{dom} \alpha[r<p \& \alpha(r)=q]]$.

The full subcategory $\mathbf{P}_{f}$ of $\mathbf{P}$ consisting of all finite posets is shown to be dually equivalent to the category of finite Brouwerian semilattices and homomorphisms. Under this duality a finite Brouwerian semilattice $\underline{A}$ corresponds with $M(\underline{A})$, the poset of all meet-irreducible elements of $A$.

The product (in $\mathbf{P}_{f}$ ) of $n$ copies ( $n \in \mathbf{N}$ ) of a one-element poset is constructed; in view of the duality this product is isomorphic to the poset of meet-irreducible elements of the free Brouwerian semilattice on $n$ generators.

If $\mathbf{V}$ is a variety of Brouwerian semilattices and if $A$ is a Brouwerian semilattice, then $A$ is $V$-critical if all proper subalgebras of $A$ belong to $\mathrm{V}$ but not $A$. It is shown that $\mathbf{a}$ variety $\mathbf{V}$ of Brouwerian semilattices has a finite equational base if and only if there are up to isomorphism only finitely many V-critical Brouwerian semilattices. This is used to show that a variety generated by a finite Brouwerian semilattice as well as the join of two finitely based varieties is finitely based. A new example of a variety without a finite equational base is exhibited.
\end{abstract}

0. Introduction. The interest in Brouwerian semilattices has been motivated mainly from two seemingly different directions. First there is intuitionistic propositional logic: The Lindenbaum algebra of its fragment $\mathcal{L}_{I C}$, which consists of formulas containing only implication and conjunction as logical connectives, is a free Brouwerian semilattice on a countable number of generators. Thus a formula is a tautology relative to $\varrho_{I C}$ if and only if it is valid in every Brouwerian semilattice. In addition the validity relation between formulas and Brouwerian semilattices sets up a Galois connection between extensions of $\mathscr{L}_{I C}$ and subvarieties of the variety of Brouwerian semilattices. Consequently algebraic methods can be successfully employed to deal with problems of a logical nature. Examples of this approach are the papers [31] and [52]; it must be said, however, that historically it was mostly full intuitionistic propositional logic (with disjunction and possibly negation) that was studied and thus on the algebraic side more interest was created in Brouwerian lattices and Heyting algebras.

On the other hand the main pioneer in the study of Brouwerian semilattices, Nemitz, considered them as algebraic objects in their own right. In [34] he initiated

Received by the editors August 22, 1980.

1980 Mathematics Subject Classification. Primary 06A12; Secondary 03B55, 03G25.

Key words and phrases. Brouwerian semilattice, duality, finitely based variety.

'This paper is the author's "Habilitationsschrift" as accepted by the Fachbereich Mathematik of the Justus-Liebig-Universität Giessen. 0002-9947/81/0000-0504/\$07.00 
an investigation of Brouwerian semilattices from a purely algebraic point of view. This has been continued by him and others, and more and more the influence of Universal Algebra as a rapidly developing research area became apparent as witnessed by the papers [40], [41] and-under a somewhat different aspect-[21]. That is, the study of Brouwerian semilattices is carried out by universal algebraic means; moreover Brouwerian semilattices enjoy many structural properties which make them readily available for examples-and counterexamples-in Universal Algebra.

Recent investigations now show a surprising convergence of these two approaches. As explained in [7] a natural premise in any attempt to study algebraic logic from the point of view of the general theory of varieties is that the varieties $\mathbf{V}$ under concern have equationally definable principal congruences in the restricted sense. And this in turn was shown in [24] to be equivalent to the assumption that for every algebra $\underline{A} \in \mathbf{V}$ the join semilattice $C p(\underline{A})$ of compact congruences is dually relatively pseudocomplemented. Due to the fact that any Brouwerian semilattice $\underline{A}$ is dually isomorphic to $\underline{C p}(\underline{A})$ the variety of Brouwerian semilattices serves as a paradigm for any 'variety of logic'. Moreover such varieties may be classified according to the variety of Brouwerian semilattices their semilattices of compact congruences generate. And this does certainly create a new stimulation to investigate the lattice of varieties of Brouwerian semilattices in more detail.

We have chosen, however, not to burden the core of this paper with any particular reference to these ideas-and likewise to those of the first approach. As a result the paper should be understood simply as a study of the category of Brouwerian semilattices from a universal algebraic point of view.

$\S 1$ introduces the notation, presents the basic definitions and contains a concise survey of the theory of Brouwerian semilattices. An attempt is made not only to quote results but also to show how some strong structural properties of the variety of Brouwerian semilattices can be most easily derived from rather simple observations. This section also introduces the universal algebraic background as far as it is needed for the investigations in the rest of the paper.

In some way $\S 2$ also has a preparatory character: Its four lemmata on meetirreducible elements and total subalgebras serve as auxiliary tools for $\$ 3$; they have been separated in order to make $\$ 3$ more readable. In addition to that some of the results of $\$ 2$ are interesting in themselves. E.g. we show that any onto homomorphism between Brouwerian semilattices preserves existing finite joins. From this we can conclude that any Brouwerian semilattice $\underline{A}$ can be imbedded into a (Brouwerian) lattice belonging to the variety generated by $A$.

$\$ 3$ is the central part of the paper. In it we develop a full duality theory for the category of finite Brouwerian semilattices. Since any finite Brouwerian semilattice is a distributive lattice it is quite clear that the objects of the dual category can be chosen to be finite partially ordered sets. Taking as morphisms partial maps which are strictly order-preserving and satisfy some additional conditions we obtain a category which we prove to be dual to the category of finite Brouwerian semilattices. Since the obvious Stone type generalization-to cover also infinite Brouwerian semilattices-did not materialize we confined ourselves to the finite case. 
This seems to be justified by the fact that this was enough to deal with the intended applications.

That is, in $\S 4$ we give a description of the posets dual to the finitely generated free Brouwerian semilattices. Unfortunately our construction can be effectively carried out only up to three generators and these cases had already been treated before in a different way. Thus the only original result concerning cardinalities is that the poset of meet-irreducible elements of the free Brouwerian semilattice on four generators has $2,494,651,862,209,437$ elements.

$\$ 5$ discusses the lattice of varieties of Brouwerian semilattices from the point of view of varieties with finite and nonfinite equational bases. It turns out that a new notion, that of a relatively critical Brouwerian semilattice, plays a crucial role: The main result of $\S 5$ is that a variety $\mathbf{V}$ of Brouwerian semilattices is finitely based if and only if there are up to isomorphism only finitely many Brouwerian semilattices critical relative to $\mathbf{V}$. This criterion is subsequently used to show that the join of finitely based varieties is again finitely based as well as to give an easy proof that a variety generated by a finite Brouwerian semilattice is finitely based. The section closes with a new example of a nonfinitely based variety.

The support of the Deutsche Forschungsgemeinschaft, which made it possible to spend a year at the University of Manitoba, is gratefully acknowledged. During this year most of the research presented in this paper was done. Thanks are due to G. Grätzer, the members of his seminar, and the other visitors at Winnipeg, in particular W. J. Blok and D. Pigozzi, for their hospitality, the constant exchange of ideas, and the atmosphere in which mathematical research thrives.

1. Preliminaries. A Brouwerian semilattice is an algebra $\underline{A}=\langle A, \wedge, *, 1\rangle$ such that the reduct $\langle A, \wedge, 1\rangle$ is a meet-semilattice with largest element 1 and where the binary operation $*$ is relative pseudocomplementation, i.e. the equivalence

$$
c \leqslant a * b \Leftrightarrow a \wedge c \leqslant b
$$

holds for all $a, b, c \in A$. For examples and the basic arithmetic of Brouwerian semilattices we refer to [34], [44]. The following rules will be frequently used without special reference. For any Brouwerian semilattice $\underline{A}$ and $a, b, c \in A$ :

$$
\begin{gathered}
1 * a=a, \\
a * a=1, \\
a * b \geqslant b, \\
a \wedge a * b=a \wedge b, \\
a *(b * c)=(a \wedge b) * c=(a * b) *(a * c), \\
a *(b \wedge c)=a * b \wedge a * c, \\
(a * b) * b \geqslant a, \\
(a * b) * b \wedge a * b=b, \\
a \leqslant b \Leftrightarrow a * b=1, \\
a \leqslant b \Rightarrow a * c \geqslant b * c \& c * a \leqslant c * b .
\end{gathered}
$$


Here we adopt the convention that * binds stronger than $\wedge$; we also use \& for the logical connective "and". Let us note that (1.3) through (1.6) may serve as an axiomatization of the class BS of all Brouwerian semilattices relative to the class of all meet-semilattices with a largest element 1. This shows that BS is an equational class, a variety in the sense of Universal Algebra. This observation should be credited to Monteiro [33].

We define a ternary polynomial $\mu$ over BS by

$$
\mu(x, y, z)=(x * y) * z \wedge(z * y) * x \wedge(z * x) * x
$$

Then it is easily checked that the identities

$$
\mu(x, z, z)=\mu(x, y, x)=\mu(z, z, x)=x
$$

hold in BS. It thus follows from a result of Pixley [43] that the variety BS has arithmetical congruences, i.e. for every $\underline{A} \in \mathbf{B S}$ the lattice $\underline{C}(\underline{A})$ of all congruence relations of $\underline{A}$ is distributive, and for any two congruence relations $\theta, \varphi$ of $\underline{A}$ we have $\theta \circ \varphi=\varphi \circ \theta$.

There is another way to see that: As known, e.g., from [34], congruence relations of a Brouwerian semilattice are in 1-1 correspondence with filters. To be more precise: If $F$ is a filter of a Brouwerian semilattice $\underline{A}$ then the relation $\theta_{F}$ with

$$
a \theta_{F} b \Leftrightarrow a * b \wedge b * a \in F
$$

is a congruence relation of $\underline{A}$. Moreover the mapping $F \rightarrow \theta_{F}$ is an isomorphism from the lattice $\underline{F}(\underline{A})$ of all filters of $\underline{A}$ onto $\underline{C}(\underline{A})$, the inverse of which is given by $\theta \rightarrow 1 / \theta$. Here $1 / \theta$ denotes the congruence class of 1 modulo $\theta$ and, similarly, we write $a / F$ for $a / \theta_{F}$ and $A / F$ for the quotient algebra $A / \theta_{F}$.

If $a, b$ are elements of a Brouwerian semilattice $\underline{A}$ then as suggested by (1.13) the filter corresponding to the principal congruence $\theta(\bar{a}, b)$ is simply the principal filter $[a * b \wedge b * a)$. It follows that for any elements $c, d \in A$ we have that

$$
c \equiv d(\theta(a, b)) \Leftrightarrow a * b \wedge b * a \leqslant c * d \wedge d * c .
$$

This means that the variety BS has equationally definable principal congruences in the restricted sense (REDPC) as mentioned in \$0. In particular, BS has the congruence extension property (CEP), i.e. if $\underline{B}$ is a subalgebra of a Brouwerian semilattice $\underline{A}$ then the natural restriction mapping maps $C(\underline{A})$ onto $C(\underline{B})$.

If $\underline{A}, \underline{B}$ are Brouwerian semilattices and if $h: A \rightarrow B$ is a homomorphism then the filter $\operatorname{ker}(h)=\{a \in A \mid h(a)=1\}$ is called the kernel of $h$. Due to the correspondence between congruence relations and filters the kernel of $h$ serves the same purpose as the congruence induced by $h$. That is, if $h$ maps $A$ onto $B$ then the Homomorphism Theorem can be stated as $\underline{A} / \operatorname{ker}(h) \simeq \underline{B}$. If, in addition, $g: A \rightarrow C$ is another homomorphism then $g$ can be factored through $h$, i.e. there exists a (unique) homomorphism $f: B \rightarrow C$ with $g=f \circ h$, if and only if $\operatorname{ker}(h) \subseteq \operatorname{ker}(g)$. In this connection let us just note that any mapping that preserves relative pseudocomplementation is already a homomorphism. This simple observation is due to Wroński [52] and, independently, Macnab [29].

A Brouwerian semilattice $\underline{A}$ is subdirectly irreducible if and only if $\underline{A}$ has a smallest nontrivial filter. This filter, however, must be a principal filter generated 
by a dual atom. I.e. $\underline{A}$ is subdirectly irreducible if and only if $A \backslash\{1\}$ has a largest element. Now if $\underline{A}$ is subdirectly irreducible and $u$ is the largest element of $A \backslash\{1\}$, then $A \backslash\{u\}$ is (the universe of) a subalgebra of $\underline{A}$. This has the consequence that $u$ must be in any generating set of $\underline{A}$. In particular, if $\underline{A}$ is generated by $n$ elements ( $n \in \mathbb{N}$ ) then $A \backslash\{u\}$ can be generated by $n-1$ elements. It follows that for fixed $n \in \mathbb{N}$ the cardinality of all $n$-generated subdirectly irreducible Brouwerian semilattices is bounded, in fact by $\left|F_{n-1}(\mathbf{B S})\right|+1$, where $F_{m}(\mathbf{B S})$ denotes the free Brouwerian semilattice on $m$ generators. Since $\left|F_{0}(\mathrm{BS})\right|=1$ we can use induction to prove that $\underline{F}_{n}$ (BS) is finite for all $n \in \mathbb{N}$, in other words the variety BS is locally finite. This argument is essentially due to Diego [13], who used it to prove that finitely generated Hilbert algebras are finite; the easy transfer to Brouwerian semilattices was first established by McKay [31]. A consequence is that the word problem (for free algebras) of BS is solvable; an explicit solution was given by Margaris [30]. See also [9] for an ALGOL program for the word problem restricted to words in three variables.

If $\pi$ is a polynomial (term) in the language of Brouwerian semilattices and $\underline{A} \in \mathbf{B S}$ we will say that $\underline{A}$ satisfies $\pi, \underline{A} \vDash \pi$, if the (polynomial) function on $\underline{A}$ induced by $\pi$ has 1 as its only value. The class of all Brouwerian semilattices satisfying $\pi$ will be denoted by $\mathbf{B S}(\pi)$. More generally, if $\Pi$ is a set of polynomials then $\mathbf{B S}(\Pi)=\{\underline{A} \in \mathbf{B S} \mid \forall \pi \in \Pi \underline{A} \vDash \pi\}$ and $\Pi$ is called an equational base for the variety $\mathbf{B S}(\Pi)$. A semantical characterization of varieties is given by Birkhoff's theorem (see e.g. [16, Theorem 3, p. 171]): A class $\mathbf{K}$ of Brouwerian semilattices is a variety if and only if $\mathbb{H}(\mathbf{K}) \subseteq K, \mathcal{S}(\mathbf{K}) \subseteq K$, and $\mathbb{P}(\mathbf{K}) \subseteq \mathbf{K}$; here $\mathbb{H}, \mathbb{S}$, $\mathbb{P}$ denote the operators of taking homomorphic images, isomorphic copies of subalgebras, and isomorphic copies of products of members of $\mathbf{K}$. If $\mathbf{K}$ is an arbitrary subclass of BS then $\mathbb{V}(\mathbf{K})=\mathbb{H S P}(\mathbf{K})$ is the smallest variety containing $\mathbf{K}$; in fact, since $\mathbf{B S}$ has the congruence extension property, the operators $\mathbb{H}$ and $S$ commute and thus also the equality $\mathbb{V}(\mathbf{K})=\operatorname{SHP}(\mathbf{K})$ holds. More important for our investigations, however, is the fact that the congruence lattices of Brouwerian semilattices are distributive. This makes Jónsson's lemma applicable for the variety BS. We will explicitly quote two of its most convenient consequences.

LEMMA 1.1 [18]. Let $\mathbf{K}$ be a finite set of finite Brouwerian semilattices and suppose that $\underline{A} \in \mathbb{V}(\mathbf{K})$ is subdirectly irreducible. Then $\underline{A} \in \mathbb{H S}(\mathbf{K})$.

This means, in particular, that if $\mathbf{V}$ is a variety generated by a finite Brouwerian semilattice then the cardinalities of subdirectly irreducible members of $\mathbf{V}$ are bounded by a finite number. Since any variety is generated by its subdirectly irreducible algebras it follows that $\mathbf{V}$ has only finitely many subvarieties.

The other consequence concerns the lattice of subvarieties of BS. Recall that the collection of all subvarieties of a given variety forms a lattice where the meet of two varieties is their intersection and their join is the variety generated by their union. To avoid set-theoretical difficulties the lattice of subvarieties of BS may be thought of as the dual of the lattice of all fully invariant congruence relations of a free Brouwerian semilattice on a countable number of generators. This also makes clear that the lattice of subvarieties of BS is distributive. 
LEMMA 1.2 [18]. Let $\mathrm{V}, \mathrm{W}$ be subvarieties of $\mathrm{BS}$ and suppose that $\underline{A}$ is subdirectly irreducible. Then $\underline{A} \in \mathbf{V} \vee \mathbf{W}$ if and oniy if $\underline{A} \in \mathbf{V}$ or $\underline{A} \in \mathbf{W}$.

These results have been the main tools used to determine the structure of the 'finite part' of the lattice of subvarieties of some congruence distributive varieties. For Brouwerian semilattices this has been done by Nemitz and Whaley [40], [41]; a fairly general treatment can be found in [11].

2. Meet-irreducible elements and total subalgebras. An element $m$ of a Brouwerian semilattice $\underline{A}$ is called meet-irreducible if $m \neq 1$ and $m=a \wedge b$ implies that $m=a$ or $m=b$. By $M(\underline{A})$ we will denote the poset of meet-irreducible elements of $\underline{A}$. The following lemma, the essence of which first appeared in [49], provides a useful alternative characterization of meet-irreducible elements.

Lemma 2.1. Let $\underline{A}$ be a Brouwerian semilattice, $m \in A \backslash\{1\}$. Then the following conditions are equivalent:

(i) $m$ is meet-irreducible.

(ii) $\forall a, b \in A[a \wedge b \leqslant m \Rightarrow a \leqslant m$ or $b \leqslant m]$.

(iii) $\forall a \in A[a * m=m$ or $a * m=1]$.

Proof. The implication (ii) $\Rightarrow$ (i) holds trivially. In order to prove that (i) implies (iii) suppose that $m$ is meet-irreducible and let $a \in A$. Then $m=a * m \wedge$ $(a * m) * m$ and thus $m=a * m$ or $m=(a * m) * m$. But the latter is easily seen to be equivalent to $a * m=1$. Finally suppose that (iii) holds. Let $a \wedge b \leqslant m$ with $a \$ m$. Then $a * m=m$ and thus $b \leqslant a * m=m$.

Next suppose that $\underline{A}$ is a Brouwerian semilattice and $X \subseteq M(\underline{A})$. Then it follows from the previous lemma that the meet-subsemilattice $B$ of $\underline{A}$ generated by $X \cup\{1\}$ is also closed under relative pseudocomplementation, in fact $B$ satisfies the stronger condition

$$
a \in A, b \in B \Rightarrow a * b \in B \text {. }
$$

Following [37] we will call subalgebras $\underline{B}$ of $\underline{A}$ satisfying (2.1) total subalgebras. More recently total subalgebras occurred-under the name Brouwerian subacts-in the theory of quasi-decompositions of Brouwerian semilattices as developed by Schmidt [45], [46]. A detailed discussion of the lattice of total subalgebras of a Brouwerian semilattice can be found in [23]. Here we confine ourselves to only those facts which we will need in subsequent sections.

First note that if $\underline{B}$ is a subalgebra of $\underline{A}$ then $m \in B$ may be meet-irreducible in $\underline{B}$ without being meet-irreducible in $\underline{A}$. The next lemma shows that this is impossible as soon as $\underline{B}$ is a total subalgebra of $\underline{A}$.

LEMMA 2.2. Let $\underline{A}$ be a Brouwerian semilattice and let $\underline{B}$ be a total subalgebra of $\underline{A}$. Then $M(\underline{B})=B \cap M(\underline{A})$.

Proof. Clearly $B \cap M(\underline{A}) \subseteq M(\underline{B})$. Let $m \in M(\underline{B})$. Then for all $a \in A$ we have that $a * m \in B$ and $(a * m) * m \in B$. Now $m=a * m \wedge(a * m) * m$ and since $m \in M(\underline{B})$ we conclude $a * m=m$ or $(a * m) * m=m$. Again this means that $a * m=m$ or $a * m=1$ and thus, by Lemma 2.1, $m \in M(\underline{A})$. 
If $A$ is a finite Brouwerian semilattice then obviously each $a \in A$ has a representation $a=\wedge M_{a}$ where $M_{a}$ is a (finite) subset of $M(\underline{A})$. By omitting some of the elements of $M_{a}$, if necessary, this representation can even be made irredundant, i.e. for all $m_{1}, m_{2} \in M_{a}$ we have $m_{1} \leqslant m_{2}$ if and only if $m_{1}=m_{2}$. In this situation we can slightly improve Lemma 2.2 .

LEMMA 2.3. Let $\underline{A}$ be a finite Brouwerian semilattice and $\underline{B}$ a total subalgebra of $\underline{A}$. If $a \in B$ and $a=\wedge M_{a}$ is an irredundant representation of $a$ with $M_{a} \subseteq M(\underline{A})$ then $M_{a} \subseteq B$.

Proof. Let $m \in M_{a}$. Then $\left(\bigwedge\left(M_{a} \backslash\{m\}\right)\right) * m=\left(\bigwedge\left(M_{a} \backslash\{m\}\right)\right) * a \in B$. If now $\wedge\left(M_{a} \backslash\{m\}\right) \leqslant m$, then by Lemma 2.1 we must have that $m_{1} \leqslant m$ for some $m_{1} \in M_{a} \backslash\{m\}$. This, however, contradicts the irredundancy. So again by Lemma 2.1 we conclude that $\bigwedge\left(M_{a} \backslash\{m\}\right) * m=m$ and thus $m \in \underline{B}$.

Next let $\underline{A}$ be a Brouwerian semilattice and $X$ a nonempty subset of $A$. Then the set

$$
A * X=\left\{\bigwedge_{i=1}^{n} a_{i} * b_{i} \mid n \in \mathbb{N}, a_{1}, \ldots, a_{n} \in A, b_{1}, \ldots, b_{n} \in X\right\}
$$

is a total subalgebra of $\underline{A}$, in fact the smallest total subalgebra containing $X$. In the case that $X$ is a subalgebra the relationship between $X$ and $A * X$ shows an additional feature: $A * X$ contains all existing joins of elements of $X$. Even more generally we prove

LEMmA 2.4. Let $\underline{A}, \underline{B}$ be Brouwerian semilattices and suppose that $h: A \rightarrow B$ is $a$ homomorphism such that $B=B * h A$. Then $h$ preserves all existing finite joins.

Proof. We restrict ourselves to prove the statement for the join of two elements $c, d \in A$. Suppose that $c \vee d$ exists; then $h(c \vee d)>h(c)$ and $h(c \vee d)>h(d)$. So let $b \in B$ such that $b \geqslant h(c), b \geqslant h(d)$. By assumption $b=\wedge_{i=1}^{n}\left(b_{i} * h\left(a_{i}\right)\right)$ for some $b_{1}, \ldots, b_{n} \in B, a_{1}, \ldots, a_{n} \in A$. Then $h(c)<b_{i} * h\left(a_{i}\right)$ for $1<i<n$ and thus $b_{i} \leqslant h(c) * h\left(a_{i}\right)=h\left(c * a_{i}\right)$. Similarly, $b_{i} \leqslant h\left(d * a_{i}\right)$ for $1 \leqslant i<n$ and, hence, $b_{i} \leqslant h\left(c * a_{i}\right) \wedge h\left(d * a_{i}\right)=h\left(c * a_{i} \wedge d * a_{i}\right)$. Consequently,

$$
\begin{aligned}
b_{i} * h\left(a_{i}\right) & \geqslant h\left(c * a_{i} \wedge d * a_{i}\right) * h\left(a_{i}\right) \\
& =h\left(\left(c * a_{i} \wedge d * a_{i}\right) * a_{i}\right) .
\end{aligned}
$$

Now observe that $\left(c * a_{i} \wedge d * a_{i}\right) * a_{i} \geqslant c \vee d$ and thus $h(c \vee d)<b_{i} * h\left(a_{i}\right)$ This finally implies that $b=\bigwedge_{i=1}^{n}\left(b_{i} * h\left(a_{i}\right)\right) \geqslant h(c \vee d)$, proving that $h(c \vee d)=$ $h(c) \vee h(d)$.

The assumptions of this lemma are certainly satisfied whenever $h$ is a homomorphism from $A$ onto $B$; thus onto homomorphisms preserve existing joins. This has an interesting consequence which is, however, a little bit out of the way: Any Brouwerian semilattice $\underline{A}$ can be imbedded into a Brouwerian lattice; the most natural candidate is the mapping $x \rightarrow(x]$ from $A$ into $J(\underline{A})$, the lattice of all lower ends of $\underline{A}$. But the example of Figure 1 shows that even if $\underline{A}$ is finite then $\underline{J}(\underline{A})$ need not belong to the variety generated by $\underline{A}$. 

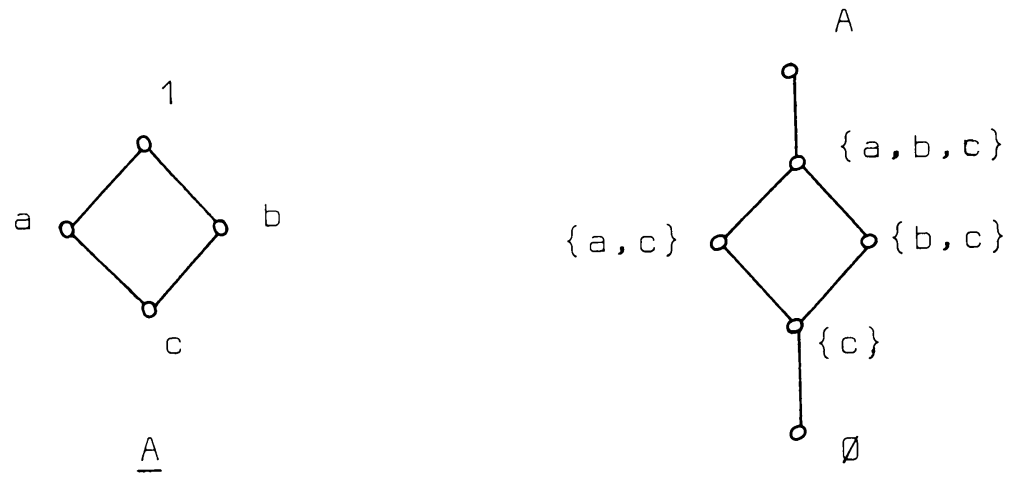

\section{Figure 1}

In contrast to this we show that every Brouwerian semilattice $\underline{A}$ can be imbedded into a Brouwerian lattice whose Brouwerian semilattice reduct belongs to the variety generated by $\underline{A}$. In fact, since BS is locally finite so is $\mathbb{V}(\underline{A})$; in particular $\mathbb{V}(\underline{A})$ is generated by its finite members. Thus $\underline{A} \in \mathbb{H S P P}(K)=\operatorname{SHP}(K)$ where $K$ consists of finite Brouwerian semilattices in $\mathbb{V}(\underline{A})$. These are all lattices, any direct product of lattices is again a lattice, and by Lemma 2.4 any image of a lattice under a Brouwerian semilattice homomorphism is a lattice. This proves our claim.

3. A duality for finite Brouwerian semilattices. Let $P$ be a poset. A subset $E \subseteq P$ is an (upper) end if $p \in E$ implies that $[p)=\{q \in P \mid q \geqslant p\} \subseteq E$. It is well known that the set $O(P)$ of all ends of $P$ is a distributive algebraic lattice. In fact, lattices of the form $O(P)$ can be characterized as those distributive algebraic lattices whose duals are algebraic too (see, e.g., [11, Proposition 1.1]). What we need here is that $O(P)$-under reverse inclusion-is a Brouwerian semilattice with operations

$$
\begin{gathered}
E \wedge F=E \cup F, \\
E * F=[F \backslash E)=\bigcup\{[p) \mid p \in F \backslash E\} .
\end{gathered}
$$

If $P$ is a finite poset then $E \in O(P)$ is meet-irreducible if and only if $E=[p)$ for some $p \in P$. This means that the mapping $p \rightarrow[p]$ is an isomorphism from $P$ onto $M(\underline{O}(P))$. On the other hand if $\underline{A}$ is a finite Brouwerian semilattice then

$$
\underline{O}(M(\underline{A})) \simeq \underline{A}
$$

where the isomorphism is given by $E \rightarrow \wedge E$. This can be considered as a variant of the well-known representation theorem for finite distributive lattices.

In the sequel we are going to extend this relationship between finite posets and finite Brouwerian semilattices to a contravariant functor from a suitable category $\mathbf{P}$ of posets to the category BS of Brouwerian semilattices.

This category $\mathbf{P}$ will be defined as follows: Its objects are posets, and its morphisms are partial mappings $\alpha: P \rightarrow Q(P, Q \in \mathbf{P})$ satisfying the conditions

$$
\forall p, r \in \operatorname{dom} \alpha[p<r \Rightarrow \alpha(p)<\alpha(r)] \text {, }
$$

$$
\forall p \in \operatorname{dom} \alpha \forall q \in Q[q<\alpha(p) \Rightarrow \exists r \in \operatorname{dom} \alpha[r<p \& \alpha(r)=q]] .
$$

(Here $\operatorname{dom} \alpha$ denotes the domain of $\alpha$.) It is easily verified that with the usual composition of partial mappings $\mathbf{P}$ is indeed a category. 
Note that condition (3.5) simply means that the image of a (principal) lower end of $P$ is again a (principal) lower end of $Q$. Thus, in particular, $\alpha P$ is a lower end of $Q$.

Now if $\alpha: P \rightarrow Q$ is a P-morphism we define $O(\alpha): O(Q) \rightarrow O(P)$ by

$$
O(\alpha)(E)=\left[\alpha^{-1} E\right) \quad(E \in O(Q)) .
$$

Since $\alpha$ is only a partial mapping, $\alpha^{-1} E$ need not be an end of $P$. Given everything as above we have

LEMMA 3.1. $O(\alpha)$ is a homomorphism of Brouwerian semilattices.

Proof. Recall that it suffices to show that $O(\alpha)$ respects relative pseudocomplementation. First note that for any subset $X$ of $Q$ we have

$$
\left[\alpha^{-1}[X)\right)=\left[\alpha^{-1} X\right) \text {. }
$$

Actually (3.7) is just an equivalent formulation of (3.5). Now let $E, F \in$ $O(Q)$. Then $O(\alpha)(E * F)=\left[\alpha^{-1}[F \backslash E)\right)$ and thus, by $(3.7), O(\alpha)(E * F)=$ $\left[\alpha^{-1}(F \backslash E)\right)$. So let $p \in O(\alpha)(E * F)$; then there exists $r \in \alpha^{-1}(F \backslash E)$ with $p \geqslant r$. In particular, $r \in \alpha^{-1} F$. If, on the other hand, $r \in\left[\alpha^{-1} E\right)$ then $r>s$ for some $s$ with $\alpha(s) \in E$. But then by (3.4) $\alpha(r) \geqslant \alpha(s)$ and this would imply $\alpha(r) \in E$, a contradiction. This shows that $r \in\left[\alpha^{-1} F\right) \backslash\left[\alpha^{-1} E\right)$ and, finally,

$$
p \in\left[\left[\alpha^{-1} F\right) \backslash\left[\alpha^{-1} E\right)\right)=O(\alpha)(E) * O(\alpha)(F) .
$$

Conversely, let $p \in O(\alpha)(E) * O(\alpha)(F)$. Then $p \geqslant r$ for some $r \in\left[\alpha^{-1} F\right) \backslash\left[\alpha^{-1} E\right)$ $\subseteq\left[\alpha^{-1} F\right) \backslash \alpha^{-1} E$. I.e. $r \geqslant s$ for some $s$ with $\alpha(s) \in F$. And if $\alpha(s) \in E$ then again by (3.4) we would have $\alpha(r) \in E$, a contradiction. Hence $s \in \alpha^{-1}(F \backslash E), p \geqslant s$ and thus $p \in O(\alpha)(E * F)$.

Clearly $O\left(1_{P}\right)=1_{O(P)}$ for any poset $P$. However, the fact that $O$ respects composition relies on (3.7). Indeed, if $P, Q, R$ are posets and $\alpha: P \rightarrow Q, \beta: Q \rightarrow R$ are $\mathbf{P}$-morphisms then by (3.7) we have that for any $E \in O(R)$,

$$
\begin{aligned}
O(\alpha)(O(\beta)(E)) & =\left[\alpha^{-1}\left[\beta^{-1} E\right)\right)=\left[\alpha^{-1} \beta^{-1} E\right) \\
& =\left[(\beta \circ \alpha)^{-1} E\right)=O(\beta \circ \alpha)(E) .
\end{aligned}
$$

Hence $O$ is a contravariant functor.

We will show that the restriction of $O$ to the full subcategory $\mathbf{P}_{f}$ of finite posets is a duality (coequivalence) between $\mathbf{P}_{f}$ and the category $\mathbf{B S}_{f}$ of finite Brouwerian semilattices. By [28, Theorem 1, p. 91] it suffices to verify that $O$ is representative, full and faithful.

That $O$ is representative has already been stated in (3.3) above. To establish the remaining properties suppose that $P, Q$ are finite posets and $h: O(Q) \rightarrow O(P)$ is a homomorphism. Then we have to show that there exists a unique P-morphism $\alpha: P \rightarrow Q$ with $h=O(\alpha)$. It is not obvious how to choose the domain of $\alpha$. An illustrating example, however, will make this clear: If $\underline{B}$ is a total subalgebra of $\underline{O}(P)$ then, by Lemma 2.2 and (3.3), $B \simeq O(Q)$ for some $Q \subseteq P$, in fact we may take $Q=\{p \in P \mid[p) \in B\}$. The corresponding $\mathbf{P}$-morphism is just the identity on 
$Q$. This suggests choosing, in general, as the domain of $\alpha$ the subposet of $P$ corresponding to the smallest total subalgebra containing the image of $O(Q)$ under $h$. By (2.2) this is $O(P) * h O(Q)$.

Now suppose that $p \in P$ is such that $[p) \in O(P) * h O(Q)$. We claim that the set $\{q \in Q \mid p \in h[q)\}$ has a largest element. To see this form the set $\mathcal{E}_{p}=\{E \in$ $O(Q) \mid p \in h(E)\}$ and put $E_{p}=\cap \mathcal{E}_{p}$. Then $E_{p} \in O(Q)$ and, by Lemma 2.4, $h\left(E_{p}\right)=\bigcap\left\{h(E) \mid E \in \mathcal{E}_{p}\right\}$. Thus $p \in h\left(E_{p}\right)$. This shows that we have for all $F \in O(Q)$,

$$
p \in h(F) \Leftrightarrow E_{p} \subseteq F .
$$

Moreover, $E_{p}=\bigcup\left\{[r) \mid r \in E_{p}\right\}$; hence $h\left(E_{p}\right)=\bigcup\left\{h[r) \mid r \in E_{p}\right\}$, which implies that $p \in h\left[r\right.$ ) for some $r \in E_{p}$. By (3.8) this means that $E_{p} \subseteq[r)$, and since $r \in E_{p}$, we conclude $E_{p}=[r)$. Consequently $r$ is the largest element of $\{q \in Q \mid p \in h[q)\}$.

Thus with $P_{1}=\{p \in P \mid[p) \in O(P) * h O(Q)\}$ we can define a mapping $\alpha: P_{1} \rightarrow Q$ by

$$
\alpha(p)=\max \{q \in Q \mid p \in h[q)\} \quad\left(p \in P_{1}\right) .
$$

Since $h: O(Q) \rightarrow O(P)$ is order-preserving we have even that for all $p \in P_{1}$, $q \in Q$,

$$
p \in h[q) \Leftrightarrow q \leqslant \alpha(p) .
$$

Also recall that $[\alpha(p))=E_{p}$; thus for all $F \in O(Q)$,

$$
p \in h(F) \Leftrightarrow \alpha(p) \in F .
$$

Everything as before,

Lemma 3.2. $\alpha$-considered as a partial mapping $\alpha: P \rightarrow Q$-is a P-morphism with $O(\alpha)=h$.

Proof. Let $p \in P_{1}$; then-since $[p)$ is meet-irreducible in $O(P)$-by $(2.2)[p)=$ $E * h(F)$ for some $E \in O(P), F \in O(Q)$. Now let $r \in P_{1}$ such that $r<p$. Clearly (3.10) shows that $\alpha(r) \leqslant \alpha(p)$. Assume that $\alpha(r)=\alpha(p)$. Then $p \in E * h(F) \subseteq$ $h(F)$ and thus by (3.11), $r \in h(F)$. Also $[p) \cup E=E * h(F) \cup E=E \cup h(F)$ and, consequently, $r \in[p) \cup E$. But if $r \in E$ then $p \in E$, which contradicts the fact that $p \in h(F) \backslash E$. On the other hand, $r \in[p)$ is impossible since $r<p$. This shows that $\alpha(r)<\alpha(p)$ and thus $\alpha$ satisfies (3.4).

Next let $p \in P_{1}$ and suppose that $q \in Q$ is such that $q<\alpha(p)$. Put $E=\{s \in$ $Q \mid s>q\}, F=[q)$. Then $E, F \in O(Q)$ and $F \backslash E=\{q\}$, i.e. $E * F=[q)$. Thus $\alpha(p) \in E * F$ and by (3.11) we have

$$
p \in h(E * F)=h(E) * h(F)=[h(F) \backslash h(E)) .
$$

Now $h(E) * h(F) \in O(P) * h O(Q), h(E) * h(F)=\bigcup\{[r) \mid r \in h(F) \backslash h(E)\}$ and thus, by Lemma 2.3, there exists $r \in P_{1}$ such that $r \in h(F) \backslash h(E)$ and $p \in[r)$. By (3.11) we conclude $\alpha(r) \in F \backslash E=\{q\}$, i.e. $r \leqslant p$ and $\alpha(r)=q$. Thus $\alpha$ also satisfies (3.5).

Finally let $E \in O(Q)$ and suppose that $p \in O(\alpha)(E)$. Then $p \in\left[\alpha^{-1} E\right)$, i.e. there exists $r \leqslant p$ such that $\alpha(r) \in E$. By (3.11) this means $r \in h(E)$ and thus also 
$p \in h(E)$. Conversely, suppose that $p \in h(E)$. As above we conclude that there exists $r \in P_{1}$ such that $r \in h(E)$ and $p \in[r)$. But then by (3.11) $\alpha(r) \in E$, i.e. $r \in \alpha^{-1} E$. This shows that $p \in\left[\alpha^{-1} E\right)=O(\alpha)(E)$. Consequently, $h=O(\alpha)$ and the proof is complete.

So far we have verified that $O$ is representative and full. Next suppose that $\beta: P \rightarrow Q$ is a P-morphism. With $h=O(\beta)$ define $\alpha$ as above. We claim that $\alpha=\beta$.

First let $p \in \operatorname{dom} \beta$. Then by (3.10) $p \in h[\beta(p))$. Moreover for $E=P \backslash(p]$ we have that $E \in O(P)$ and $p \notin E$. This shows that $p \in h[\beta(p)) \backslash E$ and hence $[p) \subseteq E * h[\beta(p))$. To prove the converse inclusion it suffices to show that $[p] \supseteq$ $h[\beta(p)) \backslash E=h[\beta(p)) \cap(p]$. So let $r \in h[\beta(p)), r \leqslant p$. Then there exists $s \in P$ such that $r \geqslant s$ and $\beta(s) \geqslant \beta(p)$. But since $s \leqslant p$ we conclude by (3.4) that also $\beta(s) \leqslant \beta(p)$ and thus, by (3.5), $s=p$. Consequently $r=p$ and so $r \in[p)$. This shows that $[p)=E * h[\beta(p))$ and thus $p \in \operatorname{dom} \alpha$.

Next suppose that $p \in \operatorname{dom} \alpha$. Then $[p)=E * h(F)$ for some $E \in O(P), F \in$ $O(Q)$. In particular, $p \in\left[\left[\beta^{-1} F\right) \backslash E\right)$, i.e. $p \geqslant r$ for some $r \in\left[\beta^{-1} F\right) \backslash E$. Again $r \geqslant s$ for some $s \in \beta^{-1} F \backslash E$ and thus $s \in \operatorname{dom} \beta$. But also $s \in E * h(F)=[p)$ and this shows that $p=s$. Hence $p \in \operatorname{dom} \beta$.

Now we can apply (3.10) to prove the equality of $\alpha$ and $\beta$. For all $p \in \operatorname{dom} \beta$ and for all $q \in Q$ we have

$$
q \leqslant \alpha(p) \Leftrightarrow p \in h[q) \Leftrightarrow p \in \beta^{-1}[q) \Leftrightarrow q \leqslant \beta(p) .
$$

This shows that $\alpha=\beta$.

As a consequence, if $\beta_{1}, \beta_{2}$ are P-morphisms such that $O\left(\beta_{1}\right)=O\left(\beta_{2}\right)$ then $\beta_{1}=\beta_{2}$, i.e. $O$ is faithful. Combining all this,

TheOrem 3.3. $O$ is a coequivalence between $\mathbf{P}_{f}$ and $\mathbf{B S}_{f}$.

One might object against this result on the grounds that the category $\mathbf{P}_{f}$ seems to be more complicated than $\mathbf{B S}_{f}$. This is certainly true; however the main advantage of working in $\mathbf{P}_{f}$ is that the objects are much less complex. E.g. a poset with 10 elements is not difficult to draw while $O(P)$ may have up to $2^{10}$ elements. Other examples given in $\S \S 4$ and 5 will substantiate this claim. Having this in mind we will close this section by characterizing subalgebras and homomorphic images of Brouwerian semilattices in terms of their associated posets (of meet-irreducible elements). The key to this is the following.

Lemma 3.4. Let $P, Q$ be posets and $\alpha: P \rightarrow Q$ a P-morphism. Then

(i) $O(\alpha)$ is 1-1 if and only if $\alpha P=Q$;

(ii) $O(\alpha)$ maps $O(Q)$ onto $O(P)$ if and only if $\alpha$ is a full imbedding, i.e. $\operatorname{dom} \alpha=P$ and $\alpha$ is 1-1.

Proof. (i) Suppose that $O(\alpha)$ is $1-1$ and let $q \in Q$. Then $O(\alpha)[q) \neq \varnothing$. Now by (3.7) $O(\alpha)[q)=\left[\alpha^{-1}[q)\right)=\left[\alpha^{-1}\{q\}\right)$. Hence $\alpha^{-1}\{q\} \neq \varnothing$ and thus $\alpha P=Q$. Conversely, suppose that $\alpha P=Q$ and let $E \in O(Q), E \neq \varnothing$. Then if $q \in E$ there exists $p \in P$ with $\alpha(p)=q$. Thus $p \in \alpha^{-1}[q) \subseteq \alpha^{-1} E$, in particular, $O(\alpha)(E)=$ $\left[\alpha^{-1} E\right) \neq \varnothing$. This shows that $O(\alpha)$ is $1-1$. 
(ii) Suppose that $O(\alpha)$ maps $O(Q)$ onto $O(P)$ and let $p \in P$. Then there exists $E \in O(Q)$ such that $[p)=O(\alpha)(E)=\left[\alpha^{-1} E\right)$. In particular, $p \in \alpha^{-1} E$, i.e. $\alpha(p)$ $\in E$. Thus $p \in \operatorname{dom} \alpha$ and hence $\operatorname{dom} \alpha=P$. Next let $p, r \in P$ such that $\alpha(p) \leqslant$ $\alpha(r)$. Let $E \in O(Q)$ be such that $[p)=O(\alpha)(E)$. Then $\alpha(p) \in E$ and hence $\alpha(r) \in E$. But then $r \in\left[\alpha^{-1} E\right)=[p)$ and this shows that $p \leqslant r$. Thus $\alpha$ is an order imbedding. Conversely, suppose that $\alpha$ is a full imbedding and let $E \in O(P)$. Put $F=\bigcup\{[\alpha(p)) \mid p \in E\}$; note that $\alpha$ is defined for every $p \in E$. Then $F \in O(Q)$; we claim that $E=O(\alpha)(F)$. So let $p \in E$; then by (3.10) $p \in \alpha^{-1}[\alpha(p))$. This shows that $E \subseteq O(\alpha)(F)$. On the other hand, let $p \in O(\alpha)(F)$; then-since $O(\alpha)$ is a homomorphism $-p \in\left[\alpha^{-1}[\alpha(r))\right)$ for some $r \in E$. Thus by (3.7) $p \in\left[\alpha^{-1}\{\alpha(r)\}\right)$, i.e. there exists $s \in P$ such that $p \geqslant s$ and $\alpha(s)=\alpha(r)$. Since $\alpha$ is 1-1 we conclude $s=r$. But then $p \geqslant r$ and thus $p \in E$. Consequently $O(\alpha)(F)=E$ and this shows that $O(\alpha)$ is onto.

Recalling that by Theorem 3.3 any homomorphism between two finite Brouwerian semilattices corresponds uniquely with a P-morphism between the respective posets of meet-irreducible elements, we obtain the announced characterization

LemMa 3.5. Let $\underline{A}, \underline{B}$ be finite Brouwerian semilattices. Then

(i) $\underline{B}$ is isomorphic to a subalgebra of $\underline{A}$ if and only if $M(\underline{B})$ is an image of $a$ subposet of $M(\underline{A})$ under a P-morphism;

(ii) $\underline{B}$ is a homomorphic image of $\underline{A}$ if and only if $M(\underline{B})$ is isomorphic to a lower end of $M(\underline{A})$.

Proof. (i) is immediately clear from Lemma 3.4(i), and (ii) is a consequence of Lemma 3.4(ii) and the remark following (3.5).

4. The free Brouwerian semilattice on $n$ generators. We are going to apply the results of the preceding section to obtain a description of the finitely generated free Brouwerian semilattices. It is immediately clear that $\underline{F}_{1}(\mathbf{B S})$ is just a two-element chain. $\underline{F}_{2}(\mathrm{BS})$ has 18 elements as first observed by Balbes [3]. Several publications treat the case of three free generators. It was de Bruijn who first determined that the exact number of elements of the free Brouwerian semilattice on three generators is $623,662,965,552,330$ [8]. His result remained partly unnoticed and was subsequently duplicated by Landolt and Whaley [27] and Krzystek [25]. For larger finite $n, F_{n}(\mathbf{B S})$ is still finite (see $\S 1$ ). A recursive construction of (the poset of meet-irreducible elements of) $F_{n}(\mathrm{BS})$ is already contained in the aforementioned paper of de Bruijn [8]. His construction is similar to the one we are going to present, his methods, however, are completely different. It should be mentioned in this context that Urquhart [51] gave a description of the free Hilbert algebra on $n$ generators; this algebra can be thought of as the set of those elements of $F_{n}(\mathbf{B S})$ which can be obtained from the free generators by repeated use of the operation * alone.

Recall that $\underline{F}_{n}(\mathrm{BS})$ is the coproduct of $n$ copies of $\underline{F}_{1}(\mathrm{BS})$ in the category BS. But since the coproduct remains finite we may consider it as the coproduct in $\mathbf{B S}_{f}$. Moreover, $\left|M\left(\underline{F}_{1}(\mathbf{B S})\right)\right|=1$ and thus, in view of the results of $\S 3$, all we have to do 
is determine the product (in $\mathbf{P}_{f}$ ) of $\boldsymbol{n}$ copies of a one-element poset. It will become clear that a general description of products in $\mathbf{P}_{f}$ which would yield a description of coproducts in $\mathbf{B S}_{f}$ seems to be extremely difficult.

So let $n$ be a natural number, $\underline{n}=\{0,1, \ldots, n-1\}$. We put $M=\{X \subseteq \underline{n} \mid X \neq$ $\varnothing\}$; for the sake of simplicity here and in the sequel our notation will not reflect the dependency on $n$. The elements of the poset $Q$ we are going to construct will be pairs $\langle X, I\rangle$ where $X$ is an element of $M$ and $I$ is a set which will contain all elements of $Q$ properly smaller than $\langle X, I\rangle$. To achieve this we define sets $S_{i}$ and mappings $f_{i}: S_{i} \rightarrow M$ recursively for all $i \in \mathbb{N}$ as follows.

$$
S_{1}=M \times\{\varnothing\}, \quad f_{1}\langle X, \varnothing\rangle=X \quad(X \in M) .
$$

(4.2) For $k>1$ :

$$
\begin{gathered}
S_{k}=\left\{\left\langle X,\left\{\left\langle Y_{1}, I_{1}\right\rangle, \ldots,\left\langle Y_{m}, I_{m}\right\rangle\right\} \cup I_{1} \cup \cdots \cup I_{m}\right\rangle \mid X \in M,\right. \\
\exists k_{1}, \ldots, k_{m}\left[\left\langle Y_{i}, I_{i}\right\rangle \in S_{k_{i}} \& \max \left\{k_{1}, \ldots, k_{m}\right\}=k-1\right. \\
\left.\left.\& X \cap \bigcup_{i=1}^{m} f_{k_{i}}\left\langle Y_{i}, I_{i}\right\rangle=\varnothing\right]\right\}, \\
\langle X, I\rangle=\left\langle X,\left\{\left\langle Y_{1}, I_{1}\right\rangle, \ldots,\left\langle Y_{m}, I_{m}\right\rangle\right\} \cup I_{1} \cup \cdots \cup I_{m}\right\rangle \in S_{k} \\
\Rightarrow f_{k}\langle X, I\rangle=X \cup \bigcup_{i=1}^{m} f_{k_{i}}\left\langle Y_{i}, I_{i}\right\rangle .
\end{gathered}
$$

Note that for $j, k \in \mathbb{N}, j \neq k$, we have $S_{j} \cap S_{k}=\varnothing$; thus with

$$
Q=\cup\left\{S_{k} \mid k \in \mathbb{N}\right\} \text {, }
$$

the union

$$
f=\cup\left\{f_{k} \mid k \in \mathbb{N}\right\}
$$

is a well-defined mapping from $Q$ into $M$. In addition, we define a mapping $g: Q \rightarrow \mathbb{N}$ by

$$
g\langle X, I\rangle=k \Leftrightarrow\langle X, I\rangle \in S_{k}
$$

Also note

$$
g\langle X, I\rangle=k \Leftrightarrow|f\langle X, I\rangle|>k .
$$

Thus $S_{n+1}=\varnothing$ and $Q=\cup_{i=1}^{n} S_{i}$; in particular, $Q$ is finite.

As already indicated we define a relation $<$ on $Q$ by

$$
\langle X, I\rangle\langle\langle Y, J\rangle \Leftrightarrow\langle X, I\rangle \in J .
$$

As an immediate consequence of (4.2) we have

$$
\langle X, I\rangle\langle\langle Y, J\rangle \Rightarrow g\langle X, I\rangle\langle g\langle Y, J\rangle
$$

as well as

$$
\langle X, I\rangle\langle\langle Y, J\rangle \Rightarrow X \cap Y=\varnothing .
$$

Thus $\langle$ is irreflexive. To prove transitivity suppose that $\langle X, I\rangle\langle\langle Y, J\rangle,\langle Y, J\rangle$ $\langle\langle Z, K\rangle$, but $\langle X, I\rangle \varangle\langle Z, K\rangle$ and $g\langle Z, K\rangle$ is minimal with respect to these 
conditions. Then $K=\left\{\left\langle Y_{1}, I_{1}\right\rangle, \ldots,\left\langle Y_{m}, I_{m}\right\rangle\right\} \cup I_{1} \cup \cdots \cup I_{m}$ for some $\left\langle Y_{1}, I_{1}\right\rangle, \ldots,\left\langle Y_{m}, I_{m}\right\rangle \in Q$. By our assumption $\langle Y, J\rangle \in K$, i.e. either $\langle Y, J\rangle$ $=\left\langle Y_{i}, I_{i}\right\rangle$ or $\langle Y, J\rangle \in I_{i}$ for some $i$ with $1 \leqslant i \leqslant m$. In the first case $\langle X, I\rangle \in J$ $=I_{i} \subseteq K$. In the second case $\langle Y, J\rangle\left\langle\left\langle Y_{i}, I_{i}\right\rangle\right.$, and by the minimality of $g\langle Z, K\rangle$ we infer that $\langle X, I\rangle\left\langle\left\langle Y_{i}, I_{i}\right\rangle\right.$. Thus $\langle X, I\rangle \in I_{i} \subseteq K$ and, hence, in both cases we arrive at a contradiction.

This shows that we can consider $Q$ as an object of $\mathbf{P}_{f}$. Now for each $i \in \underline{n}$ we define a partial mapping $\alpha_{i}: Q \rightarrow\{i\}$ by

$$
\alpha_{i}\langle X, I\rangle=i \Leftrightarrow i \in X
$$

Then (4.9) implies that $\alpha_{i}$ satisfies (3.4) and (3.5) is trivially fulfilled. Thus each $\alpha_{i}$ is a P-morphism. We claim that $Q$ (together with the $\mathbf{P}$-morphisms $\alpha_{i}, i \in n$ ) is a product of the posets $\{i\}, i \in \underline{n}$, in $\mathbf{P}_{f}$.

To prove this suppose that $R$ is a finite poset, $\beta_{i}: R \rightarrow\{i\}, i \in \underline{n}$, are P-morphisms. We have to show that there exists a unique P-morphism $\gamma: R \rightarrow Q$ such that $\alpha_{i} \circ \gamma=\beta_{i}$ for each $i \in \underline{n}$. Our proof will be accomplished by defining an appropriate mapping $h$ from $Q$ into the power set of $R$ in such a way that

$$
\gamma(r)=\langle X, I\rangle \Leftrightarrow r \in h\langle X, I\rangle
$$

holds for all $\langle X, I\rangle \in Q$. This is possible if and only if $h$ satisfies the condition

$$
\langle X, I\rangle \neq\langle Y, J\rangle \Rightarrow h\langle X, I\rangle \cap h\langle Y, J\rangle=\varnothing .
$$

That is we have to decompose $R$ into classes corresponding to the elements of $Q$ to which they will be mapped by $\gamma$. This will be done in several steps.

First let $R_{i}=\operatorname{dom} \beta_{i}$ for $i \in \underline{n}$ and $\bar{R}=\bigcup\left\{R_{i} \mid i \in \underline{n}\right\}$. Moreover, for $X \in M$ we put $R_{X}=\cap\left\{R_{i} \mid i \in X\right\} \backslash \bigcup\left\{R_{j} \mid j \notin X\right\}$. Then $\bigcup\left\{R_{X} \mid X \in M\right\}=\bar{R}$ but, more importantly,

$$
X \neq Y \Rightarrow R_{X} \cap R_{Y}=\varnothing .
$$

This decomposition of $R$ is still too coarse and we refine it by defining the mapping $h$ recursively by:

(4.14) If $g\langle X, I\rangle=1$ (i.e. $I=\varnothing$ ) then

$$
h\langle X, I\rangle=\left\{p \in R_{X} \mid \forall i \in \underline{n} \backslash X(p] \cap R_{i}=\varnothing\right\} .
$$

(4.15) If $g\langle X, I\rangle=k\rangle 1$, then

$$
\begin{aligned}
& h\langle X, I\rangle=\left\{p \in R_{X} \mid \forall\langle Y, J\rangle\right. \in I \exists r[r<p \& r \in h\langle Y, J\rangle] \\
& \&[\exists r \in \bar{R} r\langle p] \Rightarrow \exists\langle Y, J\rangle \in I r \in h\langle Y, J\rangle\} .
\end{aligned}
$$

Note that this recursive definition is possible due to (4.8). In order to prove that (4.12) holds suppose that $h\langle X, I\rangle \cap h\langle Y, J\rangle \neq \varnothing$. Then it follows from (4.13) that $X=Y$. If $I \neq J$ then we may assume that $I \neq \varnothing$, i.e. $g\langle X, I\rangle>1$ and that there is some $\langle Z, K\rangle \in I \backslash J$. Now if $p \in h\langle X, I\rangle \cap h\langle Y, J\rangle$ then by (4.15) there is some $r\langle p$ with $r \in h\langle Z, K\rangle$. But again by (4.15) there exists $\langle U, L\rangle \in J$ with $r \in h\langle U, L\rangle$. Thus $r \in h\langle Z, K\rangle \cap h\langle U, L\rangle$. By induction hypothesis $\langle Z, K\rangle=\langle U, L\rangle$ and this is a contradiction to $\langle Z, K\rangle \notin J$. Thus $I=J$ and (4.12) is proven. 
As already mentioned (4.12) guarantees that (4.11) makes $\gamma$ a well-defined partial mapping; we first show that $\gamma$ is a P-morphism. So let $p, r \in \operatorname{dom} \gamma$ be such that $p\langle r$. Let $\gamma(p)=\langle X, I\rangle, \gamma(r)=\langle Y, J\rangle$. Then $p \in h\langle X, I\rangle$ and $r \in h\langle Y, J\rangle$. Thus by (4.15) there exists $\langle Z, K\rangle \in J$ such that $p \in h\langle Z, K\rangle$. By (4.12) $\langle Z, K\rangle$ $=\langle X, I\rangle$ and thus $\langle X, I\rangle\langle\langle Y, J\rangle$. Hence $\gamma$ satisfies (3.4). Next let $p \in \operatorname{dom} \gamma$ be such that $\gamma(p)=\langle X, I\rangle$ and suppose that $\langle Y, J\rangle\langle\langle X, I\rangle$. Again by (4.15) we infer that there exists $r \in R$ such that $r\langle p$ and $r \in h\langle Y, J\rangle$. Thus $\gamma(r)=$ $\langle Y, J\rangle$ which shows that (3.5) holds for $\gamma$.

Next we claim that $\beta_{i}=\alpha_{i} \circ \gamma$ for each $i \in \underline{n}$. A basic step in proving this is to verify

$$
\bar{R}=\operatorname{dom} \gamma,
$$

which in view of (4.11) is equivalent to

$$
\bar{R}=\cup\{h\langle X, I\rangle \mid\langle X, I\rangle \in Q\}
$$

Clearly ' $\supseteq$ ' holds in (4.17) since $h\langle X, I\rangle \subseteq R_{X}$ for each $X \in M$. So let $p \in \bar{R}$. Then there exists exactly one $X \in M$ such that $p \in R_{X}$. We proceed by induction on the height of $p$ in $\bar{R}$-the height of $p$ is the maximum chain size in $(p) \cap \bar{R}$. If $p$ has height one, i.e. $p$ is a minimal element, then by (4.14) $p \in h\langle X, \varnothing\rangle$. If $p$ has bigger height then by induction hypothesis each $r \in \bar{R}$ with $r<p$ belongs to some $h\langle Y, J\rangle$. So let $I=\cup\{\{\langle Y, J\rangle\} \cup J \mid \exists r\langle p[r \in h\langle Y, J\rangle]\}$. We claim that $\langle X, I\rangle \in Q$ and $p \in h\langle X, I\rangle$. First if $r\langle p$ and $r \in h\langle Y, J\rangle$ then $X \cap f\langle Y, J\rangle$ $=\varnothing$. Indeed if $i \in X \cap f\langle Y, J\rangle$ then by (4.1) or (4.2) $i \in Z$ for some $\langle Z, K\rangle<$ $\langle Y, J\rangle$. But then by (4.15) there would exist $q\langle r$ with $q \in h\langle Z, K\rangle$. This, however, would imply that $\beta_{i}(p)=i=\beta_{i}(q)$ and $q<p$, a contradiction to the fact that $\beta_{i}$ satisfies (3.4). This shows that $\langle X, I\rangle \in Q$. Moreover it is immediately clear from the definition of $I$ that both defining conditions in (4.15) are satisfied and thus $p \in h\langle X, I\rangle$. This finishes the proof of (4.17).

Now, if $p \in R_{i}=\operatorname{dom} \beta_{i}$ then by (4.16), $p \in \operatorname{dom} \gamma$. Thus $\gamma(p)=\langle X, I\rangle$ for some $\langle X, I\rangle \in Q$, and since $p \in R_{i}$, we have $i \in X$. This shows that $\beta_{i}(p)=i=$ $\alpha_{i}\langle X, I\rangle=\alpha_{i}(\gamma(p))$. If, on the other hand, $p$ is such that $\alpha_{i}(\gamma(p))=i$ then $\gamma(p)=\langle X, I\rangle$ for some $\langle X, I\rangle \in Q$ with $i \in X$. Thus $p \in h\langle X, I\rangle \subseteq R_{X} \subseteq R_{i}$ and, hence, $\beta_{i}(p)=i=\alpha_{i}(\gamma(p))$.

It remains to show that $\gamma$ is uniquely determined. So let $\delta$ be another P-morphism such that $\alpha_{i} \circ \delta=\beta_{i}$ for each $i \in \underline{n}$. Then clearly $\bar{R} \subseteq$ dom $\delta$. On the other hand let $p \in \operatorname{dom} \delta$. Then $\delta(p)=\langle X, \bar{I}\rangle$ for some $\langle X, I\rangle \in Q$, in particular, $X \neq \varnothing$. Thus for $i \in X$ we have $i=\alpha_{i}(\delta(p))=\beta_{i}(p)$ and, hence, $p \in \bar{R}$. This shows that $\operatorname{dom} \delta=\bar{R}=\operatorname{dom} \gamma$.

Now suppose that $p \in \bar{R}$ and $\gamma(p)=\langle X, I\rangle, \delta(p)=\langle Y, J\rangle$. Then $p \in R_{X}$ and, hence, $i \in Y \Leftrightarrow \alpha_{i}(\delta(p))=i \Leftrightarrow \alpha_{i}(\gamma(p))=i \Leftrightarrow i \in X$. This shows that $Y=X$. Again we proceed by induction on the height of $p$ to show that $I=J$. If $p$ has height one then $I=\varnothing$. But if $J \neq \varnothing$ then there exists $\langle Z, K\rangle \in Q$ with $\langle Z, K\rangle<$ $\langle Y, J\rangle$. By (3.5) this implies that $\langle Z, K\rangle=\delta(r)$ for some $r\langle p$. But this contradicts the fact that $p$ has height one. Thus $J=\varnothing=I$. Now suppose that $p$ has bigger height and let $\langle Z, K\rangle \in I$. Then $\langle Z, K\rangle\langle\langle X, I\rangle=\gamma(p)$ and thus by 
(3.5) there is $r\langle p$ with $\gamma(r)=\langle Z, K\rangle$. By induction hypothesis $\delta(r)=\langle Z, K\rangle$. By (3.4) $\langle Z, K\rangle\langle\delta(p)=\langle Y, J\rangle$ and thus $\langle Z, K\rangle \in J$. The same argument shows that $\langle Z, K\rangle \in J$ implies that $\langle Z, K\rangle \in I$. Hence $I=J$.

This shows that $\gamma=\delta$ and completes the proof.

For $n=2$ the construction of $Q$ can easily be carried out. There

$$
S_{1}=\{\langle\{0\}, \varnothing\rangle,\langle\{1\}, \varnothing\rangle,\langle\{0,1\}, \varnothing\rangle\}
$$

and

$$
S_{2}=\{\langle\{0\},\{\langle\{1\}, \varnothing\rangle\}\rangle,\langle\{1\},\{\langle\{0\}, \varnothing\rangle\}\rangle\}
$$

and $Q$ has the diagram of Figure 2 .

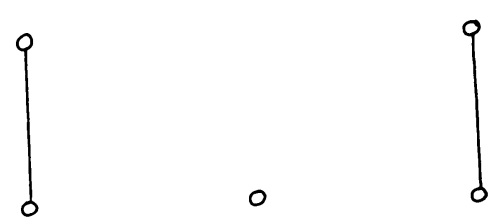

Figure 2

It is clear that already for $n=3$ an explicit listing of all the elements of $Q$ becomes messy. Figure 3 , the arrangement of which is due to de Bruijn [8], shows the poset $Q$. The labeling is chosen in such a way that with every element $\langle X, I\rangle \in Q$ we list only the elements of $X$, while $I$ can be recovered from the diagram as the set of all the elements properly smaller than $\langle X, I\rangle$.

For $n=4$ the situation is hopeless. We can, however, determine the cardinality of $Q$. To do this let us introduce the following notation: For any finite set $Z$ let $P(Z)$ be the product of the one-element posets $\{p\}, p \in Z$, as constructed before. In this sense $Q=P(n)$, in particular, $P(\varnothing)=\varnothing$. Also for any proper subset $Y$ of $\underline{n}$ let $J(P(Y))$ be the lattice of all lower ends of $P(Y)$. Now observe that for each $X \in M$ the set $\{I \mid\langle X, I\rangle \in P(\underline{n})\}$ coincides with $J(P(\underline{n} \backslash X))$. Thus we have

TheOrem 4.1. $P(\underline{n})=\{\langle X, I\rangle \mid X \in M, I \in J(P(\underline{n} \backslash X))\}$.

Note that for any poset $P$ the mapping $E \rightarrow P \backslash E$ is a bijection from $O(P)$ onto $J(P)$. This implies that in view of our duality of $\$ 3$ we have

$$
|J(P(\underline{n} \backslash X))|=|O(P(\underline{n} \backslash X))|=\left|F_{|\underline{n} \backslash X|}(\mathrm{BS})\right| .
$$

Applying this to Theorem 4.1, we get

CoROllaRY 4.2. $\left|M\left(\underline{F}_{n}(\mathbf{B S})\right)\right|=\sum_{i=0}^{n-1}\left(\begin{array}{c}n \\ i\end{array}\right)\left|F_{i}(\mathbf{B S})\right|$.

Because of $\left|F_{0}(\mathbf{B S})\right|=1,\left|F_{1}(\mathbf{B S})\right|=2,\left|F_{2}(\mathbf{B S})\right|=18$ we obtain

$$
\left|M\left(\underline{F}_{3}(\mathrm{BS})\right)\right|=1+3 \cdot 2+3 \cdot 18=61,
$$

which could have been counted directly from Figure 3. Moreover, taking from [8] that $\left|F_{3}(\mathrm{BS})\right|=623,662,965,552,330$, Corollary 4.2 yields

$$
\left|M\left(\underline{F}_{4}(\mathrm{BS})\right)\right|=2,494,651,862,209,437 .
$$

The size of $\underline{F}_{4}(\mathbf{B S})$, however, remains unknown. 


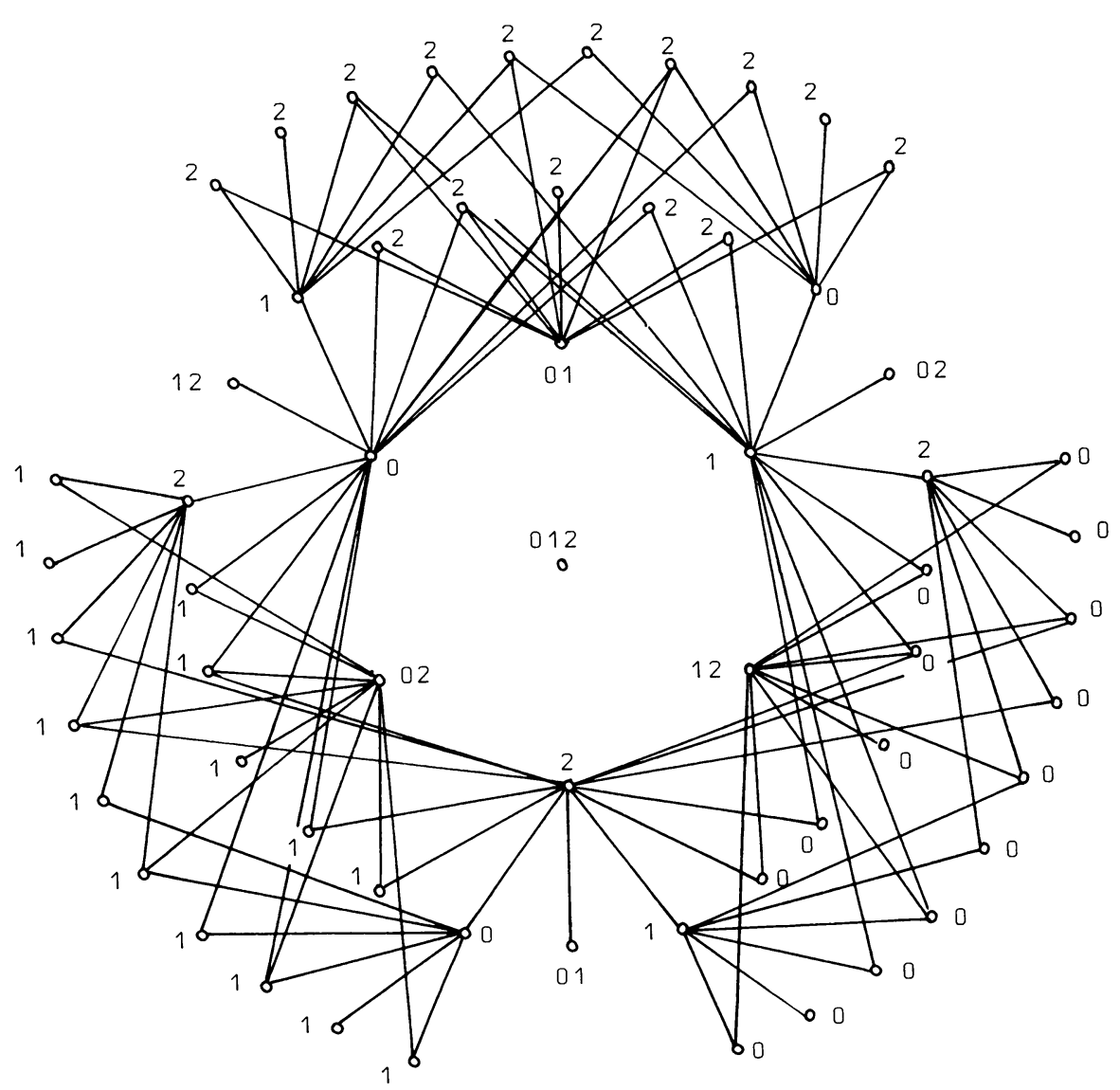

Figure 3

5. Relatively critical Brouwerian semilattices and finitely based varieties. Let $\mathbf{V}$ be a variety of Brouwerian semilattices and $\underline{A}$ a Brouwerian semilattice. $\underline{A}$ will be called $\mathbf{V}$-critical if $\underline{A} \notin \mathbf{V}$ but every proper subalgebra of $\underline{A}$ is a member of $\mathbf{V}$. This definition modifies a well-known notion from Group Theory: A group is called critical if it is finite and does not belong to the variety generated by its proper factors (see, e.g., [42, Definition 51.31]). Note that we can drop the finiteness condition since any $\mathbf{V}$-critical Brouwerian semilattice must be finite. In fact, since every algebra is contained in the variety generated by its finitely generated subalgebras, any V-critical Brouwerian semilattice has to be finitely generated and, hence, finite. Apart from that the following lemma shows that we can restrict ourselves in the definition to proper subalgebras instead of proper factors.

LEMmA 5.1. Let $\underline{A}$ be a finite Brouwerian semilattice. Then $\mathbb{H}(\underline{A}) \subseteq S(\underline{A})$.

Proof. Let $h$ be a homomorphism from $\underline{A}$ onto a Brouwerian semilattice $\underline{B}$. Then $\operatorname{ker}(h)$ is a principal filter, say $\operatorname{ker}(h)=[a)$. But then by (1.6) and (1.7) the mapping $f_{a}: A \rightarrow A$, defined by $f_{a}(b)=a * b$, is an endomorphism of $\underline{A}$ with 
$\operatorname{ker}\left(f_{a}\right)=\operatorname{ker}(h)$. Thus the Homomorphism Theorem yields that $\underline{B} \simeq f_{a} \underline{A}$, and clearly $f_{a} \underline{A} \in \mathbb{S}(\underline{A})$.

Note that this statement could also have been proven by referring to Lemma 3.5. However, as observed by Nemitz and Whaley this proof of Lemma 5.1 shows a little bit more. Namely taking $\underline{B}$ as an arbitrary finite Brouwerian semilattice and $\underline{A}$ as a free Brouwerian semilattice on a generating set of cardinality $|B|$, then clearly $\underline{A}$ admits a homomorphism onto $\underline{B}$. Now the proof of the lemma shows that $\underline{B}$ is even a retract of $\underline{A}$. Consequently every finite Brouwerian semilattice is projective in the category BS [41]. Let us just remark that the one-element Brouwerian semilattices are the only injectives.

It also follows from Lemma 5.1 that a $\mathrm{V}$-critical Brouwerian semilattice must be subdirectly irreducible. Another immediate observation is that if $\underline{A} \notin \mathrm{V}$ then $\underline{A}$ contains a $\mathbf{V}$-critical subalgebra. This means that for every proper subvariety $\mathbf{V}$ of BS there exist V-critical Brouwerian semilattices.

Let us pause for some examples. A proper subalgebra of $\underline{3}$, the three-element chain, is isomorphic to either $\underline{1}$ or $\underline{2}$. Hence $\underline{3}$ is $\mathbb{V}(\underline{2})$-critical. More generally if $\underline{A}$ is finite and subdirectly irreducible then $A$ is $\mathbf{V}$-critical, where $\mathbf{V}$ is the variety generated by the proper subalgebras of $\underline{A}$. This is a direct consequence of Lemma 1.1 .

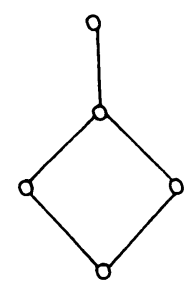

FIGURE 4

To exhibit a less trivial example consider the Brouwerian semilattice $\underline{2}^{2} \oplus 1$ which is obtained from $\underline{2}^{2}$ by adjoining a new unit (see Figure 4 ). Let $\mathbf{L}$ be the variety generated by all finite chains. It is immediately clear that any proper subalgebra of $\underline{2}^{2} \oplus \underline{1}$ is isomorphic to either of $\underline{1}, \underline{2}, \underline{3}, \underline{2}^{2}$ and hence belongs to $\mathbf{L}$. In order to prove that $\underline{2}^{2} \oplus \underline{1} \notin \mathbf{L}$ we determine an identity characterizing $\mathbf{L}$. Other identities for $\mathbf{L}$ have been found by Nemitz [35]. Also note that a reference to the general version of Jónsson's Lemma [18] could give us directly that $\underline{2}^{2} \oplus \underline{1} \notin \mathbf{L}$. Let $\lambda$ be the polynomial defined by

$$
\lambda(x, y, z)=((x * y) * z \wedge(y * x) * z) * z
$$

Then we have

$$
\text { LEMMA 5.2. } \mathbf{L}=\mathbf{B S}(\lambda) \text {. }
$$

Proof. It is clear that any chain satisfies $\lambda$. On the other hand let $\underline{A}$ be subdirectly irreducible and suppose that there are $a, b \in A$ such that neither $a<b$ nor $b \leqslant a$. Then $a * b \leqslant u$ and $b * a \leqslant u$ where $u=\max (A \backslash\{1\})$. But then $\lambda(a, b, u)=1 * u=u$ and thus $\underline{A} \not \lambda$. This shows that if $\underline{A}$ is subdirectly irreducible and $\underline{A} \vDash \lambda$ then $\underline{A}$ must be a chain. 
In particular we have seen that $\underline{2}^{2} \oplus \underline{1} \notin \mathbf{L}$ and so $\underline{2}^{2} \oplus \underline{1}$ is $\mathbf{L}$-critical. Moreover $\mathbf{L}$ is the largest variety $\mathbf{V}$ such that $\underline{2}^{2} \oplus \underline{1}$ is $\mathbf{V}$-critical. In fact, suppose that $\underline{A} \notin \mathbf{L}$. Then $\underline{A} \not \forall \lambda$ and hence there exist elements $a, b, c \in A$ such that $\lambda(a, b, c) \neq 1$. But then a straightforward calculation shows that the elements $a * b \wedge b * a, a * b$, $b * a, \lambda(a, b, c), 1$ are all distinct and form a subalgebra of $\underline{A}$ which is isomorphic to $\underline{2}^{2} \oplus 1$. In other words, $\underline{A} \in \mathbf{L}$ if and only if $\underline{2}^{2} \oplus \underline{1} \notin S(\underline{A})$. This is an instance of a well-known phenomenon. A variety $\mathbf{V}$ of Brouwerian semilattices is called a splitting variety if it splits the lattice of all subvarieties of $\mathbf{B S}$ into two disjoint intervals, i.e. there exists a variety $\mathbf{W}$ such that for all varieties $\mathbf{U}$ we have either $\mathbf{U} \subseteq \mathbf{V}$ or $\mathbf{W} \subseteq \mathbf{U}$. In this sense $\mathbf{L}$ is a splitting variety, its 'partner' is the variety $\mathbb{V}\left(\underline{2}^{2} \oplus \underline{1}\right)$.

The notion of a splitting variety had first been introduced by McKenzie in the context of lattice varieties [32] and has since then been a powerful tool in the study of the lattice of subvarieties of some varieties such as of lattices, Heyting algebras [12], modal algebras [5], [6] and even Brouwerian semilattices [41].

Now, if $\mathbf{V}$ is a splitting variety of Brouwerian semilattices then the corresponding variety $\mathbf{W}$ can be generated by a finite subdirectly irreducible Brouwerian semilattice. To see this suppose that $A \in \mathrm{W}$ is finite and subdirectly irreducible. If $\mathbb{V}(\underline{A}) \subset \mathbf{W}$ then $\mathbb{V}(\underline{A}) \subseteq \mathbf{V}$. But since $\mathbf{W} \nsubseteq \mathbf{V}$ there must be some finite subdirectly irreducible $\underline{A}$ such that $\mathrm{W}=\mathbb{V}(\underline{A})$.

Conversely every finite subdirectly irreducible Brouwerian semilattice gives rise to a splitting variety as already observed by Nemitz and Whaley [41]. It follows easily from Lemma 5.1 that for any finite subdirectly irreducible Brouwerian semilattice $\underline{A}$ the class

$$
\text { BS: } \underline{A}=\{\underline{B} \in \mathbf{B S} \mid \underline{A} \notin S(\underline{B})\}
$$

is a variety, in fact the splitting variety corresponding with $\mathbb{V}(\underline{A})$. Moreover, following an idea of Jankov [17], we can even exhibit an identity defining BS: $\underline{A}$.

To do this suppose that $\underline{A}$ is finite and subdirectly irreducible, $|A|=n \in \mathbb{N}$. We may thus assume that $A=\{1, \ldots, n\}$ where the labeling is chosen in such a way that $1_{\underline{A}}=1$ and 2 is the unique dual atom of $\underline{A}$. Now we define polynomials $\delta_{A}, \varepsilon_{A}$ by

$$
\begin{gathered}
\delta_{A}\left(x_{1}, \ldots, x_{n}\right)=\bigwedge_{1<i, j<n}\left(\left(x_{i} * x_{j}\right) * x_{i * j} \wedge x_{i * j} *\left(x_{i} * x_{j}\right)\right), \\
\varepsilon_{A}\left(x_{1}, \ldots, x_{n}\right)=\delta_{\underline{A}}\left(x_{1}, \ldots, x_{n}\right) * x_{2} .
\end{gathered}
$$

The obvious valuation shows that $\underline{A} \sharp \varepsilon_{A}$. If, on the other hand, $\underline{B}$ is a Brouwerian semilattice such that $\underline{B} \forall \varepsilon_{\underline{A}}$ then there exist $b_{1}, \ldots, b_{n} \in B$ with $\varepsilon_{A}\left(b_{1}, \ldots, b_{n}\right) \neq$ 1. Note that for $1 \leqslant \bar{i}, j \leqslant n$ we have $\left(b_{i} * b_{j}\right) * b_{i * j} \wedge b_{i * j} *\left(b_{i} * b_{j}\right) \geqslant$ $\delta_{A}\left(b_{1}, \ldots, b_{n}\right)$ and thus, by $(1.10)$ and $(1.6), \delta_{\Lambda}\left(b_{1}, \ldots, b_{n}\right) * b_{i * j}=$ $\delta_{A}\left(b_{1}, \ldots, b_{n}\right) *\left(b_{i} * b_{j}\right)$. This shows that the mapping $f: A \rightarrow B$ defined by $f(i)=$ $\delta_{A}\left(b_{1}, \ldots, b_{n}\right) * b_{i}$ is a homomorphism. Moreover $f(2)=\varepsilon_{A}\left(b_{1}, \ldots, b_{n}\right) \neq 1$, and since 2 is the unique dual atom of $\underline{A}$ we conclude that $f$ is $1-1$. This shows that $\underline{A} \in S(\underline{B})$. Together with the fact that $\underline{A} \not \varepsilon_{\underline{A}}$ this means that

$$
\text { BS: } \underline{A}=\mathbf{B S}\left(\varepsilon_{A}\right) \text {. }
$$


In particular it follows that every splitting variety has a finite equational base. And since the union of equational bases of varieties is a base for their intersection we see that any finite intersection of splitting varieties is finitely based. We will extend this observation to a characterization of finitely based varieties of Brouwerian semilattices.

To facilitate notation suppose that $S$ is a set which contains exactly one member of each isomorphism class of finite subdirectly irreducible Brouwerian semilattices. For a variety $\mathbf{V}$ let $C(\mathbf{V})=\{\underline{A} \in S \mid \underline{A}$ is $\mathbf{V}$-critical $\}$. We have seen before that if $\mathbf{V} \neq \mathbf{B S}$ then $C(\mathbf{V}) \neq \varnothing$. More importantly,

LEMmA 5.3. Let $\mathbf{V}$ be a subvariety of BS. Then $\mathrm{V}=\cap_{\underline{A} \in C(\mathrm{~V})}$ BS: $\underline{A}$.

Proof. Let us denote the right-hand side by $\mathbf{W}$. Then $\underline{A} \in C(\mathbf{V})$ implies that $\underline{A} \notin \mathbf{V}$ and thus $\mathbf{V} \subseteq \mathbf{B S}: \underline{A}$. This gives $\mathbf{V} \subseteq \mathbf{W}$. If now $\mathbf{V} \subset \mathbf{W}$ then there exists $\underline{A} \in \mathbf{W} \backslash \mathbf{V}$. Hence we find a $\mathbf{V}$-critical subalgebra $\underline{B}$ of $\underline{A}$. Now $\underline{B} \simeq \underline{C}$ for some $\underline{C} \in C(\mathbf{V})$. But then $\underline{C} \notin \mathbf{B S}: \underline{C}$ and thus $\underline{C} \notin W$. This is a contradiction since $\underline{C} \in \mathbb{S}(\underline{A}) \subseteq W$.

Moreover, this representation of $\mathbf{V}$ as an intersection of splitting varieties is minimal in the following sense. If $X \subseteq C(\mathbf{V})$ and $\mathbf{V}=\cap_{A \in X} \mathbf{B S}: \underline{A}$ then $X=$ $C(\mathbf{V})$. Indeed let $\underline{B} \in C(\mathbf{V})$, then $\underline{B} \notin \mathrm{V}$ and thus $\underline{B} \notin \mathbf{B S}: \underline{A}$ for some $\underline{A} \in X$. Hence $\underline{A} \in S(\underline{B})$ and, since $\underline{B}$ is V-critical, we must have $\underline{A}=\underline{B}$.

Now assume that $\mathbf{V}$ is a finitely based variety. Then $\mathbf{V}$ is a dually compact element in the lattice of subvarieties of BS. This together with Lemma 5.3 implies that $\mathbf{V}=\bigcap_{A \in X} \mathbf{B S}: \underline{A}$ for some finite subset $X$ of $C(\mathbf{V})$. But then the preceding remarks show that actually $X=C(\mathbf{V})$, i.e. $C(\mathbf{V})$ is finite. All this amounts to

THEOREM 5.4. A variety $\mathbf{V}$ of Brouwerian semilattices is finitely based if and only if there exist (up to isomorphism) only finitely many V-critical Brouwerian semilattices.

The advantage of this criterion is that we can use semantical methods rather than syntactical ones in order to determine whether a variety is finitely based or not. We are going to demonstrate this in the following when we prove that a variety generated by a finite Brouwerian semilattice is finitely based. This result does follow from the 'Finite Basis Theorem' of Baker [1] which states that any congruence-distributive variety which is generated by a finite algebra has a finite equational base. In our case, however, we can give an easy proof resting on Theorem 5.4.

THEOREM 5.5. Let $\underline{A}$ be a finite Brouwerian semilattice. Then $\mathbb{V}(\underline{A})$ is finitely based.

Proof. Clearly the statement is true if $|A|=1$. Thus we may assume that $|A| \geqslant 2$. Let $\underline{B}$ be $\mathbb{V}(\underline{A})$-critical. Then $|B| \geqslant 3$ and there is a proper subdirectly irreducible subalgebra $\underline{C}$ of $\underline{B}$ which is maximal among all (subdirectly irreducible) subalgebras containing the dual atom of $\underline{B}$. Since $\underline{B}$ is $\mathbb{V}(\underline{A})$-critical we have $\underline{C} \in \mathbb{V}(\underline{A})$ and thus by Lemmas 1.1 and $5.1, \underline{C} \in \mathbb{S}(\underline{A})$. In particular, $|C| \leqslant|A|$. Now, if $b \in B \backslash C$ then the subalgebra [ $C \cup\{b\}]$ generated by $C \cup\{b\}$ is still 
subdirectly irreducible, hence $[C \cup\{b\}]=B$. Thus $\underline{B}$ has a generating set of cardinality $\leqslant|A|+1$. And since BS is locally finite there are up to isomorphism only finitely many $\mathbb{V}(\underline{A})$-critical Brouwerian semilattices.

A similar argument can be used to prove the following.

THEOREM 5.6. Let $\mathrm{V}, \mathrm{W}$ be finitely based subvarieties of BS. The $\mathrm{V} \vee \mathrm{W}$ is finitely based.

Proof. By Lemma 5.3 we have $\mathrm{V}=\cap_{\underline{A} \in C(\mathrm{~V})}$ BS: $\underline{A}, \mathbf{W}=\cap_{\underline{B} \in C(\mathrm{~W})}$ BS: $\underline{B}$. Now the lattice of subvarieties of BS is distributive and thus

$$
\mathbf{V} \vee \mathbf{W}=\bigcap_{\langle\underline{A}, \underline{B}\rangle \in C(\mathbf{V}) \times C(\mathbf{W})}(\mathbf{B S}: \underline{A} \vee \mathbf{B S}: \underline{B}) .
$$

Thus it suffices to show that if $\underline{A}$ is $\mathrm{V}$-critical and $\underline{B}$ is W-critical then BS: $\underline{A} \mathrm{~V}$ BS: $\underline{B}$ is finitely based. Moreover we may assume that BS: $\underline{A}$ and BS: $\underline{B}$ are incomparable or, equivalently, $\underline{A} \in \mathbf{B S}: \underline{B}$ and $\underline{B} \in \mathbf{B S}: \underline{A}$. Now suppose that $\underline{C}$ is (BS: $\underline{A} \vee$ BS: $\underline{B}$ )-critical. Then $\underline{C} \notin$ BS: $\underline{A}$ and $\underline{C} \notin \mathrm{BS}: \underline{B}$, i.e. $\underline{A} \in \mathcal{S}(\underline{C})$ and $\underline{B} \in \widehat{S}(\underline{C})$. We may even assume that $A \subseteq \bar{C}$ and $B \subseteq C$. Let $D=[\bar{A} \cup B \cup\{u\}]$, where $u$ is the dual atom of $\underline{C}$. Then $\underline{D}$ is subdirectly irreducible, $\underline{A} \in \mathbb{S}(\underline{D})$, $\underline{B} \in \mathcal{S}(\underline{D})$. Hence $\underline{D} \notin \mathrm{BS}: \underline{A}$ and $\underline{D} \notin \mathrm{BS}: \underline{B}$ and thus, by Lemma $1.2, \underline{D} \notin \mathrm{BS}$ : $\underline{A} \vee$ BS: $\underline{B}$. Since $\underline{C}$ is (BS: $\underline{A} \vee \mathbf{B S}: \underline{B}$ )-critical we conclude that $D=C$. Consequently $\underline{C}$ has a generating set of cardinality $\leqslant|A|+|B|+1$. As above, this proves the claim.

Here, however, we have to admit that there is an easy syntactical proof which rests on an almost obvious generalization of an idea of Nemitz and Whaley [41, Lemma 4.4].

First let $\pi$ be an $m$-ary and $\rho$ an $n$-ary polynomial. Then clearly $B S(\pi, \rho)=$ $B S(\sigma)$ where $\sigma$ is the $(m+n)$-ary polynomial defined by $\sigma\left(x_{1}, \ldots, x_{m+n}\right)=$ $\pi\left(x_{1}, \ldots, x_{m}\right) \wedge \rho\left(x_{m+1}, \ldots, x_{m+n}\right)$. Consequently any finitely based variety of Brouwerian semilattices is one-based. Note that this could have been concluded directly from the fact that BS, and hence every subvariety of $\mathbf{B S}$, is arithmetical. Now it is not difficult to check that $B S(\pi) \vee B S(\rho)=B S(\tau)$ where $\tau$ is the $(m+n+1)$-ary polynomial defined by

$$
\begin{aligned}
\tau\left(x_{1}, \ldots, x_{m+n+1}\right)= & \left(\left(\left(\pi\left(x_{1}, \ldots, x_{m}\right) * x_{m+1}\right) * x_{m+1}\right) * \rho\left(x_{m+2}, \ldots, x_{m+n+1}\right)\right) \\
& * \rho\left(x_{m+2}, \ldots, x_{m+n+1}\right) .
\end{aligned}
$$

In any case the finitely based subvarieties of BS form a sublattice of the lattice of varieties of Brouwerian semilattices. In other words, it is impossible to construct nonfinitely-based varieties from 'nice' varieties using only finitary lattice operations. In the following we will present an example of a variety which does not have a finite equational base. The first such example was given by Wroński [52].

For $n \in \mathbb{N}$ let $P_{n}$ be the poset of Figure 5. We claim that the set $\left\{\underline{O}\left(P_{n}\right) \mid n \in \mathbb{N}\right\}$ is a strictly independent set of subdirectly irreducible Brouwerian semilattices, i.e. they satisfy

$$
\forall n, m \in \mathbb{N}\left[\underline{O}\left(P_{n}\right) \in \mathbb{S}\left(\underline{O}\left(P_{m}\right)\right) \Leftrightarrow n=m\right]
$$



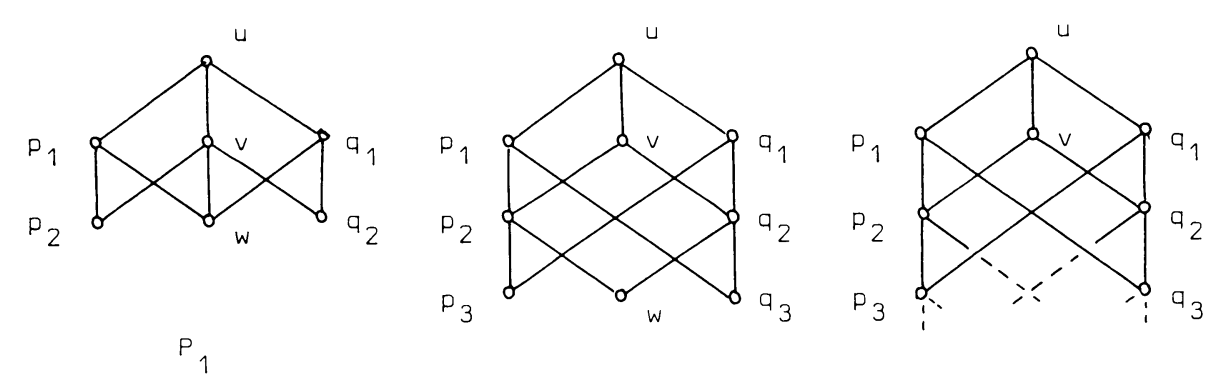

$P_{2}$

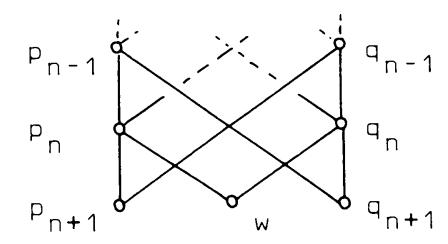

Figure 5

It follows then that for every $n \in \mathbb{N}, \underline{O}\left(P_{n}\right)$ is V-critical where

$$
\mathbf{V}=\bigcap_{n \in N} \mathbf{B S}: \underline{O}\left(P_{n}\right)
$$

In fact, $\underline{O}\left(P_{n}\right) \notin V$ and if $\underline{A}$ is a proper subalgebra of $\underline{O}\left(P_{n}\right)$ then $\underline{A} \in$ BS: $\underline{O}\left(P_{n}\right)$ as well as $\underline{A} \in$ BS: $\underline{O}\left(P_{m}\right)$ for $m \neq n$, since otherwise $\underline{O}\left(P_{m}\right) \in \mathbb{S}(\underline{A}) \subseteq \mathbb{S}\left(\underline{O}\left(P_{n}\right)\right)$. Thus by Theorem $5.4 \mathrm{~V}$ cannot have a finite equational base.

To prove that (5.6) holds we use Lemma 3.5(i): So let $n, m \in \mathbb{N}$ and suppose that $\alpha$ is a $\mathbf{P}$-morphism from $P_{m}$ onto $P_{n}$. Note that then necessarily $m \geqslant n$. Now let $s_{1}, s_{2}, s_{3} \in P_{m}$ such that $\alpha\left(s_{1}\right)=p_{1}, \alpha\left(s_{2}\right)=q_{1}, \alpha\left(s_{3}\right)=v$. It follows from (3.4) that $s_{1}, s_{2}, s_{3}$ must be pairwise incomparable. Moreover $\left(p_{1}\right] \neq\left\{p_{1}\right\}$ and thus (3.5) implies that $\left\{s_{1}, s_{2}, s_{3}\right\}=\left\{p_{1}, q_{1}, v\right\}$. First suppose that $\alpha(v) \neq v$, we may assume that $\alpha(v)=p_{1}, \alpha\left(p_{1}\right)=v, \alpha\left(q_{1}\right)=q_{1}$. Now $q_{2}<q_{1}$; thus by (3.5) there exists $t \in P_{m}$ such that $t<q_{1}$ and $\alpha(t)=q_{2}$. Note that $q_{2} \Varangle p_{1}$ and hence, by (3.4), $t \nless v$. This, however, is impossible. We conclude that $\alpha(v)=v$ and again we may assume that $\alpha\left(p_{1}\right)=p_{1}$ and $\alpha\left(q_{1}\right)=q_{1}$. Next by (3.5) there must be $t \in P_{m}, t<p_{1}$, such that $\alpha(t)=p_{2}$, again $t \nless q_{1}$ and thus $t=p_{2}$. Similarly we prove $\alpha\left(q_{2}\right)=q_{2}$ and, by way of induction, $\alpha\left(p_{i}\right)=p_{i}, \alpha\left(q_{i}\right)=q_{i}$ for $i \leqslant n+1$. Now $w<p_{n}$ and thus, by (3.5), there exists $t \in P_{m}, t<p_{n}$, with $\alpha(t)=w$. Again since $w \nless p_{n+1}$ we must have that $t \nless p_{n+1}$. If now $m>n$ then there are only two elements $t \in P_{m}$ satisfying these properties, namely $p_{n+1}$ and $q_{n+2}$. But since $\alpha\left(p_{n+1}\right)=p_{n+1} \neq w$ we are left with $\alpha\left(q_{n+2}\right)=w$. This, however, is impossible since $q_{n+2}<q_{n+1}$ and by (3.4) this implies that $w=\alpha\left(q_{n+2}\right)<\alpha\left(q_{n+1}\right)=q_{n+1}$. This shows that $n=m$ and completes the proof of (5.6).

Just as in Wroński's example we can draw one more conclusion from (5.6).

THEOREM 5.7. BS has $2^{\kappa_{0}}$ subvarieties. 
Proof. It is well known that BS (and likewise any variety with only finitary operations) has at most $2^{\kappa_{0}}$ subvarieties. On the other hand we associate with each $M \subseteq \mathbb{N}$ the variety $\mathbf{U}_{M}=\bigcap_{n \in M}$ BS: $\underline{O}\left(P_{n}\right)$. Then it follows from (5.6) that for $M_{1} \neq M_{2}$ the varieties $U_{M_{1}}$ and $U_{M_{2}}$ are distinct. Since $\mathbb{N}$ has $2^{\boldsymbol{N}_{0}}$ subsets this proves the result.

\section{BIBLIOGRAPHY}

1. K. A. Baker, Finite equational bases for finite algebras in a congruence-distributive equational class, Adv. in Math. 24 (1977), 207-243.

2. R. Balbes, $A$ representation theory for prime and implicative semilattices, Trans. Amer. Math. Soc. 136 (1969), 261-267.

3. On free pseudo-complemented and relatively pseudo-complemented semi-lattices, Fund. Math. 78 (1973), 119-131.

4. R. Balbes and Ph. Dwinger, Distributive lattices, Univ. of Missouri Press, Columbia, Mo., 1974.

5. W. J. Blok, Varieties of interior algebras, Ph.D. thesis, Univ. of Amsterdam, 1976.

6. The lattice of modal logics; an algebraic investigation, J. Symbolic Logic 45 (1980), 221-236.

7. W. J. Blok and D. Pigozzi, The deduction theorem in algebraic logic (manuscript).

8. N. G. de Bruijn, Exact finite models for minimal propositional calculus over a finite alphabeth, T.H.-Report 75-WSK-02, Dept. of Math., Eindhoven Technological University, 1975.

9. An ALGOL-program for deciding derivability in minimal propositional calculus with implication and conjunction over a three letter alphabeth, Memorandum 1975-06, Dept. of Math., Eindhoven Technological University, 1975.

10. The use of partially ordered sets for the study of non-classical propositional logics, Problèmes Combinatoires et Théorie des Graphes, Colloques Internat. C.N.R.S., No. 260, 1978, pp. 67-70.

11. B. A. Davey, On the lattice of subvarieties, Houston J. Math. 5 (1979), 183-192.

12. A. Day, Varieties of Heyting algebras (unpublished manuscript).

13. A. Diego, Sur les algèbres de Hilbert, Collection de Logique Mathématique, Ser. A, No. 21, Paris, 1966.

14. J. Eastham and W. C. Nemitz, Density and closure in implicative semilattices, Algebra Universalis 5 (1975), 1-7.

15. O. Frink, Pseudo-complements in semi-lattices, Duke Math. J. 29 (1962), 505-514.

16. G. Grätzer, Universal algebra, 2nd ed., Springer-Verlag, New York and Berlin, 1979.

17. V. A. Jankov, The relationship between deducibility in the intuitionistic propositional calculus and finite implicational structures, Soviet Math. Dokl. 4 (1963), 1203-1204.

18. B. Jónsson, Algebras whose congruence lattices are distributive, Math. Scand. 21 (1967), 110-121.

19. T. Katriňák, Die Kennzeichnung der distributiven pseudokomplementären Halbverbände, J. Reine Angew. Math. 241 (1970), 160-179.

20. __ Remarks on the W. C. Nemitz's paper “Semi-Boolean Lattices", Notre Dame J. Formal Logic 11 (1970), 425-430.

21. P. Köhler, The semigroup of varieties of Brouwerian semilattices, Trans. Amer. Math. Soc. 241 (1978), 331-342.

22. ___ Endomorphism semigroups of Brouwerian semilattices, Semigroup Forum 15 (1978), 229-234.

23. __ Brouwerian semilattices: The lattice of total subalgebras, Algebra and Its Applications, Banach Center Publications (to appear).

24. P. Köhler and D. Pigozzi, Varieties with equationally definable principal congruences, Algebra Universalis 11 (1980), 213-219.

25. P. S. Krzystek, On the free relatively pseudocomplemented semilattice with three generators, Rep. Math. Logic 9 (1977), 31-38.

26. W. J. Landolt and T. P. Whaley, Relatively free implicative semi-lattices, Algebra Universalis 4 (1974), 166-184.

27. $\ldots$ The free implicative semi-lattice on three generators, Algebra Universalis 6 (1976), 73-80.

28. S. Mac Lane, Categories for the working mathematician, Springer-Verlag, New York and Berlin, 1971. 
29. D. S. Macnab, An algebraic study of modal operators on Heyting algebras with applications to topology and sheafification, Ph. D. thesis, Univ. of Aberdeen, 1976.

30. A. Margaris, Identities in implicative semilattices, Proc. Amer. Math. Soc. 41 (1973), 443-448.

31. G. McKay, The decidability of certain intermediate propositional logics, J. Symbolic Logic 33 (1968), 258-264.

32. R. McKenzie, Equational bases and nonmodular lattice varieties, Trans. Amer. Math. Soc. 174 (1972), 1-43.

33. A. Monteiro, Axiomes indépendants pour les algèbres de Brouwer, Rev. Un. Mat. Argentina 17 (1955), 149-160.

34. W. C. Nemitz, Implicative semi-lattices, Trans. Amer. Math. Soc. 117 (1965), 128-142.

35. __ Semi-Boolean lattices, Notre Dame J. Formal Logic 10 (1969), 235-238.

36. __ On the lattice of filters of an implicative semilattice, J. Math. Mech. 18 (1969), 683-688.

37. _ Implicative homomorphisms with finite range, Proc. Amer. Math. Soc. 33 (1972), 319-322.

38. __ Implicative semi-lattices and ternary algebras, Manuscripta Math. 29 (1973), 39-41.

39. __ Irreducibility in implicative semilattices, Algebra Universalis 9 (1979), 244-249.

40. W. C. Nemitz and T. Whaley, Varieties of implicative semilattices, Pacific J. Math. 37 (1971), 759-769.

41. ___ Varieties of implicative semi-lattices. II, Pacific J. Math. 45 (1973), 303-311.

42. H. Neumann, Varieties of groups, Springer-Verlag, New York and Berlin, 1967.

43. A. F. Pixley, Distributivity and permutability of congruence relations in equational classes of algebras, Proc. Amer. Math. Soc. 14 (1963), 105-109.

44. H. Rasiowa, An algebraic approach to non-classical logics, North-Holland, Amsterdam, 1974.

45. J. Schmidt, Quasi-decompositions, exact sequences and triple sums of semigroups. I. General theory, Contributions to Universal Algebra, Szeged, Colloq. Math. Soc. János Bolyai 17 (1975), 365-397.

46. __ Quasi-decompositions, exact sequences and triple sums of semigroups. II. Applications, Contributions to Universal Algebra, Szeged, Colloq. Math. Soc. János Bolyai 17 (1975), 399-428.

47. , Binomial pairs, semi-Brouwerian and Brouwerian semilattices, Notre Dame J. Formal Logic 19 (1978), 421-434.

48. J. Schmidt and C. Tsinakis, Relative pseudo-complements, join-extensions and meet-retractions, Math. Z. 157 (1977), 271-284.

49. D. Smith, Meet-irreducible elements in implicative lattices, Proc. Amer. Math. Soc. 34 (1972), $57-62$.

50. C. Tsinakis, Brouwerian semilattices determined by their endomorphism semigroups, Houston J. Math. 5 (1979), 427-436.

51. A. Urquhart, Implicational formulas in intuitionistic logic, J. Symbolic Logic 39 (1974), 661-664.

52. A. Wronski, The degree of completeness of some fragments of the intuitionistic propositional calculus, Rep. Math. Logic 2 (1974), 55-61.

Mathematisches Institut, Justus-Liebig-Universitït Giessen, 6300 Giessen, Federal Republic OF GERMANY 